\title{
CONTINUITY OF THE EQUILIBRIUM PRICE DENSITY AND ITS USES IN PEAK-LOAD PRICING
}

\author{
Anthony Horsley and Andrew J Wrobel \\ London School of Economics and Political Science
}

Contents:

Abstract

1. Introduction

2. Peak-Load Pricing with Cross-Price Independent Demands

3. Peak-Load Pricing with Storage and Independent Demands

4. Commodity and Price Spaces for a General Framework

5. Symmetry and Weaker Conditions on Production Sets

6. Sub-Symmetry of Preferences

7. Continuity of the Equilibrium Price Density Function

8. Continuity and Boundedness of Price and Quantity with Additively Separable Utility

9. Application to Peak-Load Pricing with Storage 10. Conclusions

Appendix A - Lower and Upper Essential Values

Appendix B - Uniform Continuity and Inverse Continuity

References

Discussion paper

No.TE/01/417

May 2001
The Suntory Centre

Suntory and Toyota International Centres for Economics and Related Disciplines London School of Economics and Political Science Houghton Street London WC2A 2AE

Tel.: 020-7955 6698 


\begin{abstract}
With $L^{\infty}$ as the commodity space, the equilibrium price density is shown to be a continuous function of the commodity characteristics. The result is based on symmetry ideas from the Hardy-Littlewood-Pólya theory of rearrangements; and it includes, but is not limited to, the case of symmetric (rearrangementinvariant) production costs and additively separable consumer utility. For continuous-time peak-load pricing of, e.g., electricity, this allows the inclusion of storage and of cross-price dependent demands. In this context a continuously varying price has two uses. First, it excludes the demand jumps that arise from discontinuous switches from one price rate to another. Second, in the operation and valuation of hydroelectric and pumped-storage plants (studied elsewhere), price continuity guarantees that their capacities (viz., the reservoir and the converter), the energy stocks and, in the case of hydro also the river flows, have well-defined marginal values.
\end{abstract}

Keywords: Price density; continuous-time peak-load pricing; pumped storage.

JEL Nos.: D51, D58, L94.

(C) by the authors. All rights reserved. Short sections of text, not to exceed two paragraphs, may be quoted without explicit permission, provided that full credit, including (C) notice, is given to the source.

Contact address: Dr Anthony Horsley, Department of Economics, London School of Economics and Political Science, Houghton Street, London WC2A 2AE. E-mail: LSEecon123@msn.com 


\section{Introduction}

With cyclically priced goods such as electricity, a discontinuous time-of-use (TOU) tariff is likely to result in disequilibrium by causing demand discontinuities that are incompatible with pricing at marginal cost: e.g., with a sudden price drop, any consequential demand jump means that the marginal cost also increases and begins to differ from the ruling price. In such circumstances an equilibrium price has, therefore, to be a continuous function of time. For the case of cross-price independent demand for electricity generated from a thermal technology, this result can be obtained by the elementary method of supply and demand curves, the intersection of which varies continuously in time if the demand curve does (Section 2). By exploiting the results of [16], given also in [11], the argument can be extended to include energy storage (Section 3). This is useful because, as we show in [16], price continuity guarantees that the capacities of a storage plant (viz., the reservoir and the converter) have definite marginal values, i.e., the short-run profit function is differentiable in the fixed inputs. ${ }^{1}$ These values are instrumental in the short-run approach to long-run equilibrium based on our extension of the Wong-Viner Envelope Theorem [15].

With purely thermal electricity generation (and with outages assumed away) the SR supply curve is constant over the cycle, represented by the interval $[0,1]$. So- on the assumption of no start-up or shutdown costs - the SR cost, as well as the LR cost, is a symmetric (a.k.a. rearrangement-invariant) function of the output bundle $(y(t))_{t \in[0,1]}$. This means that the production cost, $C(y)$, depends on the values of $y$ but not on their particular arrangement on $[0,1]$. In the language of electricity suppliers, the cost is a function of the load-duration curve, which mathematically is the decreasing rearrangement of $y$ (Definition 5.5). Cost symmetry can serve as a basis for a more general method of proving price continuity in an equilibrium. This uses a strong implication of cost symmetry, viz., the similarity of arrangement between the trajectories of output $y$ and of the supporting price $p$ (Lemma 5.9 with Remark 5.10), which means that it cannot be that $p\left(t^{\prime}\right)<p\left(t^{\prime \prime}\right)$ and $y\left(t^{\prime}\right)>y\left(t^{\prime \prime}\right)$. With symmetry, this holds globally, i.e., for any instants $t^{\prime}$ and $t^{\prime \prime}$ in $[0,1]$.

A local and weaker condition formalises the notion that a price jump cannot entail a drop in supply (Definition 5.11). On the demand side, a slightly stronger condition means that a price jump must entail a drop in demand (Definition 6.1). Together, these conditions rule out a price jump in equilibrium (Theorem 7.2). The assumption on consumer demand captures more than just the case of additively separable utility, and thus extends beyond the case of independent demands (Example 6.3). On the production side, the assumption generalises the symmetric and the additively separable cases; and it is weakened further to include technologies such as energy storage (Definition 5.14 and Lemma 9.1). In this context our general framework improves on the method of curves because it applies also with interdependent demands.

\footnotetext{
${ }^{1}$ For the profit to be differentiable, the price does not have to be a pure density; it suffices that the density part be continuous [11]. This is useful when the price also contains a "singular" part (see below).
} 
Although it is motivated by a continuous-time problem, this analysis applies to a good differentiated by any commodity characteristic that ranges over a topological space $T$ which carries an "underlying" measure $\sigma$. (For example, $\sigma$ could be a probability on a "continuum" of events.) In such a context, quantities of goods and their values are integrals with respect to (w.r.t.) $\sigma$, and the commodity space must therefore consist of functions, from $T$ into the real line $\mathbb{R}$, that are integrable, square-integrable or bounded (depending on the problem); and it must be paired with a suitable price space. A pair of Lebesgue spaces, $L^{\rho}$ and $L^{\rho^{\prime}}$ with $(1 / \rho)+\left(1 / \rho^{\prime}\right)=1$, is an example; and the price space $L^{\rho^{\prime}}$ is the norm-dual of $L^{\rho}$ when $\rho<+\infty$.

For peak-load pricing the relevant case is that of $\rho=+\infty$ : the functions representing commodity bundles must be bounded because the problem involves capacity costs or constraints. The norm-dual of $L^{\infty}[0,1]$ is larger than $L^{1}[0,1]$, and Bewley $\left[1\right.$, p. 516] asserts that the elements of $L^{\infty *} \backslash L^{1}$ "have no economic interpretation". This is a mistaken view because these so-called "price singularities" have an essential role as capacity charges when the output $y \in L^{\infty}[0,1]$ has a pointed peak: if the set $\{t: y(t)=\operatorname{Sup}(y)\}$ has zero Lebesgue measure then the subdifferential $\partial \operatorname{Sup}(y)$ lies wholly in $L^{\infty *} \backslash L^{1}$. When the equilibrium allocation actually lies in the smaller commodity space of continuous $\mathbb{R}$-valued functions, $\mathcal{C}[0,1]$, such a price functional can be restricted to $\mathcal{C}$ and represented by a singular measure. Thus it acquires a tractable mathematical form and can be used as part of a TOU tariff. For example, when the demand for electricity has a firm pointed peak, a point measure represents the capacity charge in $\$$ per $\mathrm{kW}$ demanded at the peak instant, whilst the fuel charge is a price density in $\$ / \mathrm{kWh}$ : see [10].

The type of equilibrium which Bewley's price space $L^{1}[0,1]$ does accommodate is one with the capacity charge spread as a density over a peak plateau in the output. Such an equilibrium arises if the users' utility and production functions are Mackey continuous (which means that consumption is interruptible, i.e., that a brief interruption causes only a small loss of utility or output): see [12]. For this case we identify the conditions on which the equilibrium price function, $p^{\star}$, is not only integrable but also continuous (or, more precisely, has a continuous variant).

Our price continuity result consists, then, in using conditions specific to Lebesgue function spaces (viz., symmetry, additive separability and their generalisations) to show that the price function's essential limit exists everywhere on $T$-in which case it is "automatically" continuous on $T$. Thus we combine ideas from the theory of rearrangements (for functions) with the concept of the essential limit (known from the theory of stochastic processes, where it is used to prove the continuity of sample paths). This argument is quite different from the usual approach to price representation, which relies on topological assumptions on preferences and production sets in a commodity space paired with the "target" price space. When the price space is $\mathcal{C}(T)$, this approach yields an equilibrium existence result for the commodity space of measures $\mathcal{M}(T)$, obtained by Jones [18]. This is of interest in commodity differentiation, but it does not apply directly to a good which comes as a flow and can only be 
consumed over time. In such a case an integrable or bounded function can represent a consumption bundle, but a point measure cannot (since it would mean consuming a positive amount in literally no time, i.e., at an infinite rate). It might seem that there is the possibility of indirect application to the commodity space $L^{\infty}[0,1]$ by embedding it in $\mathcal{M}[0,1]$. But even without production, this requires an extension of preferences from their original domain $L_{+}^{\infty}[0,1]$ to $\mathcal{M}_{+}[0,1]$. This is obviously impossible in the case of a strictly concave, additively separable utility, because such a function is not continuous for the weak topology $\mathrm{w}\left(L^{\infty}, L^{1}\right)$, and a fortiori it can have no w $(\mathcal{M}, \mathcal{C})$-continuous extension (since $\left.\mathcal{C} \subset L^{1}\right)$ : see, e.g., $\left[1\right.$, p. 539]. ${ }^{2}$ As Jones shows by means of a one-consumer example without production [18, Example 4], the equilibrium price density can be discontinuous also when the utility $U$, on $L_{+}^{\infty}[0,1]$, is additively separable and the marginal instantaneous utility, at a constant consumption rate, is continuous or even constant, i.e., when $U(x)=\int_{0}^{1} u(x(t)) \mathrm{d} t$. He concludes that in "models of commodity differentiation...the computational convenience of additively separable preferences must be abandoned" [18, p. 525]. However, when $L^{\infty}[0,1]$ is the commodity space, this merely means that, with such preferences, price continuity cannot be established by embedding $L^{\infty}$ in $\mathcal{M}$. Jones' counterexample rests on the discontinuity of the initial endowment $\omega$, and it does not contradict our analysis. What it does show is that the embedding approach remains ineffective with any other choice of the topology on $\mathcal{M}$ that makes $\mathcal{C}$ the continuous dual; the strongest is the Mackey topology $\mathrm{m}(\mathcal{M}, \mathcal{C})$. Although a general framework such as Richard's [21, Case 1] applies whenever the preferences are continuous and uniformly proper w.r.t. $\omega,^{3}$ these assumptions can only fail with extensions of the additively separable utility $U{ }^{4}$

Since the method of curves does not require a formal vector-space framework, it is presented first (Sections 2 and 3). Commodity and price spaces are introduced in Section 4. This is followed by a discussion of symmetry and its generalisations, in Sections 5 and 6 . Section 7 gives the general price continuity theorem. The case of additively separable utility (without cost separability) is spelt out in Section 8 (with additional results which show that both price and quantity trajectories are continuous and bounded). The application to electricity pricing with storage (which extends and

\footnotetext{
${ }^{2}$ Jones $\left[18\right.$, p. 525] also points out that an extendable utility $U_{r}$ is obtained by averaging the instantaneous consumption over a small $r$-neighbourhood of each $t$. However, price continuity for such a case is rather obvious because the "moving average" $(1 / 2 r) \int_{t-r}^{t+r} p(\tau) \mathrm{d} \tau$ is always continuous in $t$, for every integrable $p$.

${ }^{3}$ Richard's Case 2 does not apply to the commodity space $\mathcal{M}[0,1]$ because no topology makes it a locally convex (topological) vector lattice.

${ }^{4}$ Even if $U$ had an $\mathrm{m}(\mathcal{M}, \mathcal{C})$-continuous extension to $\mathcal{M}_{+}[0,1]$, it could not be $\omega^{\prime}$-uniformly proper w.r.t. any $\omega^{\prime}$, for if it were, it would also be so w.r.t. any $\omega^{\prime \prime} \geq \omega^{\prime}$, and by Richard's result this would imply the existence of an equilibrium price $p^{\star} \in \mathcal{C}$ with $\omega^{\prime \prime}$ as the endowment. But if $\omega^{\prime \prime}$ is chosen to be discontinuous on $[0,1]$ as in Jones' example, then $p^{\star}(t)=(\mathrm{d} u / \mathrm{dx})\left(\omega^{\prime \prime}(t)\right)$ is also discontinuous. (It is also obvious that $U$ cannot have a continuous extension to all of $\mathcal{M}$, since if it did then $\nabla U(x)$ would always be in $\mathcal{C}$, which is not the case when $x$ is discontinuous.)
} 
supersedes Section 3) comes in Section 9. Appendix A reviews the concept of essential value (or limit). Appendix B reviews some properties of continuous functions.

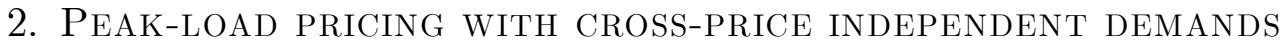

The simplest model of equilibrium consists of supply and demand curves $S$ and $D$ in the price-quantity plane; and if one or both curves vary continuously with a parameter such as time, then so does their intersection point. This observation is useful in continuous-time peak-load pricing, i.e., pricing a cyclically demanded good which is produced by one or more techniques with capacity costs (in addition to variable costs). In this context a continuous price can serve as an equilibrium solution to the problem of demand jumps caused by discontinuous switches from one price rate to another in a TOU tariff. A local demand maximum arises on the wrong side of such an instant, viz., just after a price drop (or just before a price jump). For example, the introduction of a two-rate tariff usually results in a surge of demand just after the switch from the daytime rate to the night-time rate: see, e.g., [20, pp. 65-66 with Figure 2.2]. Since this is a typical example, the cyclically priced flow in question is henceforth referred to as electricity, although the model applies to other goods as well.

With a one-station technology, the long-run marginal cost (LRMC) tariff has the form $p_{\mathrm{LR}}^{\star}(t)=w+r \gamma^{\star}(t)$, where $r$ is the unit capacity cost, $w$ is the unit running cost, and $\gamma^{\star}$ (with $\int \gamma^{\star}(t) \mathrm{d} t=1$ ) is the distribution of the capacity charge, which is concentrated on the (global) maxima of the LR equilibrium output $y_{\mathrm{LR}}^{\star}$. To keep demand constant during the peak, the price varies continuously with time. With a multi-station technology the tariff structure is more complex: the offpeak price varies between the lowest and the highest of the unit variable costs of the various station types. Marginal cost pricing means that the offpeak price is the generating system's marginal variable cost, i.e., the unit fuel cost of the marginal station on line. (In the long run the system must also be optimal, i.e., it must minimise the total cost of meeting the demand.) Thus the marginalist principle might appear to imply discontinuous price changes: with, say, a two-station technology with variable costs $w_{1}<w_{2}$ it seems that the price must drop from $w_{2}$ to $w_{1}$ as soon as the demand (at price $w_{2}$ ) has fallen to $k_{1}$, the capacity of the first, base type. But the users' response to such a sudden price drop is likely to reverse, albeit temporarily, the downward trend of demand - in which case, to meet the demand at the price $w_{1}$, the second station must immediately be switched back on, and the marginal fuel cost increases back to $w_{2}$. This undermines the tariff because the ruling price, $w_{1}$, differs from the marginal cost. And if the tariff is revised to take account of the new demand trajectory, new price discontinuities are created, so the difficulty arises afresh. As we show, there is nevertheless an equilibrium solution which consists in lowering the price gradually, from $w_{2}$ to $w_{1}$, to keep the demand constant and equal to $k_{1}$ for a time after the peak station has been switched off. The price keeps falling just enough to maintain the demand (which would fall below $k_{1}$ if the price were kept constant at $w_{2}$ ). After the 
transition period the price "freezes" at $w_{1}$, and demand starts falling again. Price and quantity move alternately (along the vertical and horizontal segments of the supply curve in Figure 1a), i.e., the price and output trajectories have alternating plateaux: see Figures $1 \mathrm{~b}$ and $1 \mathrm{~d} .^{5}$ Thus price continuity implies that, in addition to the peak plateau, the output has offpeak plateaux during which the price changes from $w_{2}$ to $w_{1}$ and vice versa.

In practice a continuous price change could be approximated by a number of small price jumps. A cruder but effective device is to stagger a price drop by timing it differently for different consumers. For example, since 1977 Electricité de France has spread the onset of its night-time rate over one and a half hours; ${ }^{6}$ each consumer is notified of his particular night period but is given no choice in the matter. Since the effect on market demand is akin to facing the "average consumer" with a price varying between the two rates, this can be viewed as a rough implementation, workable even with two-rate or three-rate meters, of the exact pure pricing solution.

With a cross-price independent demand for electricity and a purely thermal generating technology, the method of supply and demand curves applies directly to the SR equilibrium, and it extends to the LR equilibrium by the SR approach. The perfectly competitive SR supply curve depends on the generating capacities $\left(k_{\theta}\right)$ and their unit running costs $\left(w_{\theta}\right)$, where $\theta=1, \ldots, \Theta$ are the various station types. If the current electricity price is $\mathrm{p}$, then the supply from station type $\theta$ is: $S_{\theta}(\mathrm{p})=0$ for $\mathrm{p}<w_{\theta}$, $S_{\theta}(\mathrm{p})=k_{\theta}$ for $\mathrm{p}>w_{\theta}$, and $S_{\theta}(\mathrm{p})=\left[0, k_{\theta}\right]$ for $\mathrm{p}=w_{\theta}$ (in which case $S_{\theta}(\mathrm{p})$ is multivalued). The total supply is $S_{\mathrm{Th}}(\mathrm{p})=\sum_{\theta=1}^{\Theta} S_{\theta}(\mathrm{p})$. For, say, $\Theta=2$ with $w_{1}<w_{2}$, the total supply from a two-station thermal system $k=\left(k_{1}, k_{2}\right)$ is

$$
S_{\mathrm{Th}}(\mathrm{p})= \begin{cases}0 & \text { for } \mathrm{p}<w_{1} \\ {\left[0, k_{1}\right]} & \text { for } \mathrm{p}=w_{1} \\ k_{1} & \text { if } w_{1}<\mathrm{p}<w_{2} \\ {\left[k_{1}, k_{1}+k_{2}\right]} & \text { for } \mathrm{p}=w_{2} \\ k_{1}+k_{2} & \text { for } \mathrm{p}>w_{2}\end{cases}
$$

(Figure 1a). This is, of course, the supply schedule of a producer whose SR cost is additively separable over the cycle $[0, T]$; here

$$
C_{\mathrm{SR}}(y(\cdot))=\int_{0}^{T} c_{\mathrm{SR}}(y(t)) \mathrm{d} t
$$

\footnotetext{
${ }^{5}$ The graphs in Figures $1 \mathrm{~b}$ and $1 \mathrm{~d}$ are not periodic. They can be thought of in two ways: either as representing only a part of the cycle, or as representing the whole cycle, but after a rearrangement of time which produces nonincreasing price- and load-duration curves (and which exists by Lemma 5.9 and Remark 5.7).

${ }^{6}$ With a uniformly timed night-time rate, the EdF's experience in 1976 was that demand would surge, just after the start of the low rate, by over $3 \mathrm{GW}$ (ca. $7 \%$ of maximum demand).
} 
with $c_{\mathrm{SR}}(\mathrm{y})=\int_{0}^{\mathrm{y}} S_{\mathrm{Th}}^{-1}(\mathrm{y})$ dy (Figure 1c). The demand $D_{t}(\mathrm{p})$ is, at any time $t$, a function of the current price alone. It can be interpreted as the demand of a household maximising the utility function

$$
U(x(\cdot), m)=m+\int_{0}^{T} u(t, x(t)) \mathrm{d} t
$$

over $x(\cdot) \geq 0$ and $m \geq 0$ subject to the budget constraint $m+\int_{0}^{T} p(t) x(t) \mathrm{d} t \leq M$, where $M$ is the income and $p(\cdot)$ is a TOU price in terms of the numeraire (which represents all other goods and thus closes the model). With this behaviour, the equilibrium price can be expressed in terms of marginal utility and thus shown to be continuous in $t$ if $\partial u / \partial \mathbf{x}$ is continuous. For each $t$ the instantaneous utility $u(t, \mathrm{x})$ is taken to be a strictly concave, increasing and differentiable function of the consumption rate $\mathbf{x} \in \mathbb{R}_{+}$, with $(\partial u / \partial \mathbf{x})(t, 0)>w_{1}$ (to ensure that the SR equilibrium demand is positive at every $t$, if $\left.k_{1}>0\right)$. For simplicity all demand is assumed to come from a single household. Its income $M$ is the sum of an endowment of the numeraire $\left(m^{\text {En }}\right)$ and the pure profit from electricity sales, i.e.,

$$
M=m^{\mathrm{En}}+\sum_{\theta=1}^{2}\left(\int_{0}^{T}\left(p(t)-w_{\theta}\right)^{+} \mathrm{d} t-r_{\theta}\right) \cdot k_{\theta}
$$

where $r_{1}$ and $r_{2}$ are the unit capacity costs (per cycle), and $\pi^{+}=\max \{\pi, 0\}$ is the nonnegative part of $\pi$. To guarantee a positive demand for the numeraire, assume that $m^{\mathrm{En}}>\sum_{\theta=1}^{2}\left(T w_{\theta}+r_{\theta}\right) k_{\theta}$. Then at any time $t$ the demand depends only on the current price $p(t)$, and it is determined from the equation

$$
\frac{\partial u}{\partial \mathbf{x}}(t, x(t))=p(t)
$$

(In other words, $D_{t}(\mathbf{p})=((\partial u / \partial \mathbf{x})(t, \cdot))^{-1}(\mathbf{p})$.) When $w_{2} \leq(\partial u / \partial \mathbf{x})\left(t, k_{1}+k_{2}\right)$, this value of $\partial u / \partial \mathrm{x}$ is the price needed to equate demand to $k_{1}+k_{2}$. Similarly, when $w_{1} \leq(\partial u / \partial \mathbf{x})\left(t, k_{1}\right) \leq w_{2}$, the middle term is the price needed to equate demand to $k_{1}$. So the SR equilibrium price can be given as

$$
p_{\mathrm{SR}}^{\star}(t)=w_{1}+\min \left\{w_{2}-w_{1},\left(\frac{\partial u}{\partial \mathrm{x}}\left(t, k_{1}\right)-w_{1}\right)^{+}\right\}+\left(\frac{\partial u}{\partial \mathrm{x}}\left(t, k_{1}+k_{2}\right)-w_{2}\right)^{+}
$$

which is continuous in $t$ if $\partial u / \partial \mathbf{x}$ is (for any fixed $\mathbf{x}>0$ ). If additionally $w_{1}>$ $\min _{t}(\partial u / \partial \mathbf{x})\left(t, k_{1}\right)$ and $w_{2}<\max _{t}(\partial u / \partial \mathbf{x})\left(t, k_{1}\right)$, then the times $t$ with $p_{\mathrm{SR}}^{\star}(t)$ between $w_{1}$ and $w_{2}$ (and with the equilibrium output equal to $k_{1}$ ) form a set of positive measure. ${ }^{7}$ With $k_{2}>0$, this is an offpeak plateau in the output (Figure 1d).

\footnotetext{
${ }^{7}$ Note that meas $\left\{t: w_{1}<(\partial u / \partial \mathbf{x})\left(t, k_{1}\right)<w_{2}\right\}>0$ because this set is nonempty and open.
} 
The LR equilibrium is obtained from the SR equilibrium by solving the simultaneous equations $r_{\theta}=\int_{0}^{T}\left(p_{\mathrm{SR}}^{\star}\left(t, k_{1}, k_{2}\right)-w_{\theta}\right)^{+} \mathrm{d} t$ for $k$ and putting the solution $k^{\star}$ into $p_{\mathrm{SR}}^{\star}(t, k) .^{8}$
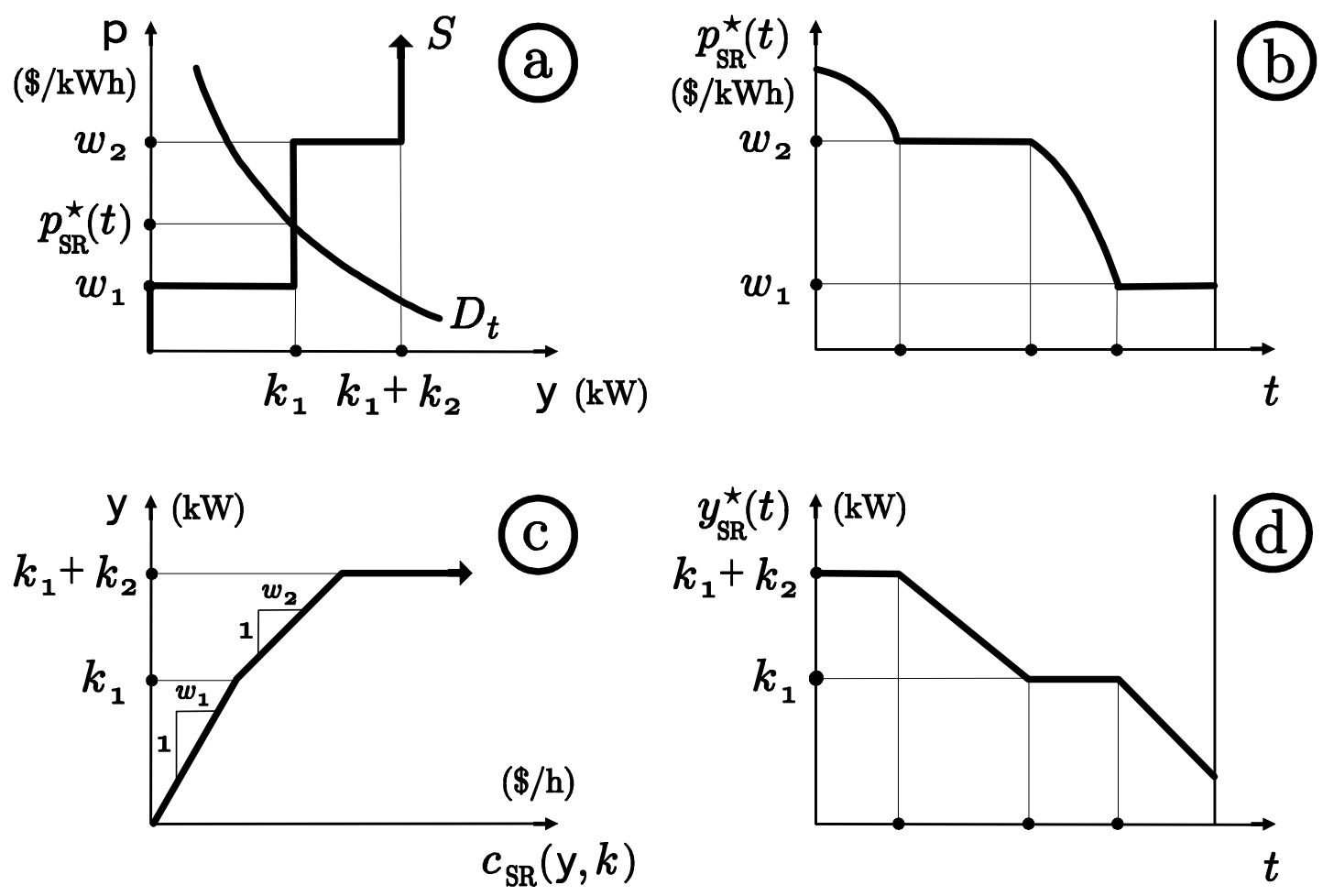

FiguRE 1. Short-run equilibrium of supply and (cross-price independent) demand for thermally generated electricity: (a) determination of the price and output for each instant $t$; (b) and (d) the trajectories of price and output; (c) the SR cost curve (the integral of $S$ w.r.t. y).

The SR price formula (2.3) extends to the case of any number, $\Theta$, of stations. Also, no inequalities between the $w_{\theta}$ 's need be assumed. This is useful when $w_{\theta}$ depends on time, and there may be no fixed merit order among the stations. Denote by $w^{\uparrow} \in \mathbb{R}^{\Theta}$ the nondecreasing rearrangement of the vector $w=\left(w_{\theta}\right)_{\theta=1}^{\Theta} \in \mathbb{R}^{\Theta}$ (i.e., $w_{1}^{\uparrow}$ is the smallest entry in $w, w_{2}^{\uparrow}$ is the second smallest, and so on). In these terms

$$
p_{\mathrm{SR}}^{\star}(t)=w_{1}^{\uparrow}(t)+\sum_{\theta=1}^{\Theta-1} \min \left\{\left(w_{\theta+1}^{\uparrow}(t)-w_{\theta}^{\uparrow}(t)\right),\left(\frac{\partial u}{\partial \mathrm{x}}\left(t, \sum_{\alpha: w_{\alpha}(t) \leq w_{\theta}^{\uparrow}(t)} k_{\alpha}\right)-w_{\theta}^{\uparrow}(t)\right)^{+}\right\}
$$

\footnotetext{
${ }^{8}$ In the case of a corner solution with $k_{2}=0$, only the inequality $r_{2} \geq \int_{0}^{T}\left(p_{\mathrm{SR}}^{\star}-w_{2}\right)^{+} \mathrm{d} t$ holds.
} 


$$
+\left(\frac{\partial u}{\partial \mathbf{x}}\left(t, \sum_{\theta=1}^{\Theta} k_{\theta}\right)-w_{\Theta}^{\uparrow}(t)\right)^{+}
$$

\section{Peak-LOAd PRicing With Storage And indePEndent Demands}

In addition to eliminating demand jumps, price continuity is useful for the problem of operating and valuing storage facilities for cyclically priced goods. In the context of electricity this applies to hydroelectric and pumped-storage plants. Here we deal with pumped storage (PS); the case of hydro is similar. Unlike a thermal plant, a storage plant has two capital inputs, viz., the reservoir capacity $k_{\mathrm{St}}$ (in $\mathrm{kWh}$ ) and the conversion capacity $k_{\mathrm{Co}}$ (in $\mathrm{kW}$ ) which transforms the stored energy into electricity and vice versa. (For a more detailed description of the technology, see [16] and Section 9 here.) On the basis of operating-profit maximisation, given a TOU electricity price $p$ and the plant's capacities $k_{\mathrm{PS}}=\left(k_{\mathrm{St}}, k_{\mathrm{Co}}\right)$, the stock of energy can be assigned a TOU shadow price $\psi(t)$, which — as we show in [16] - is unique if $p(t)$ is continuous in $t$. In general there is a set of such stock prices $\hat{\Psi}\left(p, k_{\mathrm{PS}}\right)$, but it has just one element, $\hat{\psi}\left(p, k_{\mathrm{PS}}\right)$, if $p$ is continuous. It then follows that the capacities, and in the case of hydro also the river flow river flow ([13] and [14]), have definite and separate marginal values. This underlines the importance of price continuity in general equilibrium.

In terms of any $\psi \in \hat{\Psi}\left(p, k_{\mathrm{PS}}\right)$, the storage plant's optimal output rate $y(t)$ can be given as in (3.1) below: $y(t)= \pm k_{\mathrm{Co}}$ if $p(t) \neq \psi(t)$, with $y(t) \in\left[-k_{\mathrm{Co}}, k_{\mathrm{Co}}\right]$ if $p(t)=\psi(t)$. For each $t$ this defines the plant's supply curve $S_{\mathrm{PS}, t}$ in the price-quantity plane, but the curve is not cross-price independent because it depends on $\psi(t)$, which depends on the whole function $p$. This means that, with a combined generation and storage system, the SR equilibrium price cannot be found by intersecting curves as in the purely thermal case. Nevertheless, if $p_{\mathrm{SR}}^{\star}$ is an equilibrium tariff, and $S_{t}$ is the system's supply curve constructed from $k_{\theta}, w_{\theta}, k_{\mathrm{Co}}$ and a $\psi \in \hat{\Psi}\left(p_{\mathrm{SR}}^{\star}, k_{\mathrm{PS}}\right)$, then-for a certain choice, $\psi_{\mathrm{SR}}^{\star}$, of $\psi$ - the curve $S_{t}$ intersects the demand curve $D_{t}$ at $p_{\mathrm{SR}}^{\star}(t)$. This fact can still be used to show that $p_{\mathrm{SR}}^{\star}$ is continuous, although the argument requires an extra step, which is to show that $\psi_{\mathrm{SR}}^{\star}(t)$ is continuous in $t$ (and hence that $S_{t}$ varies continuously with $t$ ). Once $\psi_{\mathrm{SR}}^{\star}$ is known to be continuous, continuity of $p_{\mathrm{SR}}^{\star}$ follows, as in the purely thermal case, from (2.4), which is now applied with $\Theta+1$ instead of $\Theta$ and with $w_{\Theta+1}(t):=\psi_{\mathrm{SR}}^{\star}(t)$. (It also follows that $\psi_{\mathrm{SR}}^{\star}=\hat{\psi}\left(p_{\mathrm{SR}}^{\star}\right)$.)

Continuity of $\psi_{\mathrm{SR}}^{\star}$ is next deduced from equilibrium conditions and two properties of every $\psi \in \hat{\Psi}$, viz., (i) that $\psi$ is of bounded variation, so it has the two one-sided limits $\psi(t \pm),{ }^{9}$ and (ii) that $\psi$ rises or falls (possibly with a jump or a drop) only when the reservoir is full or empty, respectively. Consider a system with, say, two thermal stations as in (2.1) and one storage station (with conversion capacity $k_{\mathrm{Co}}$ ).

\footnotetext{
${ }^{9}$ Since the set of $t$ 's with $\psi(t-) \neq \psi(t+)$ is at most countable, it does not matter which of the two values is chosen for $\psi(t)$ itself.
} 
With $p_{\mathrm{SR}}^{\star}$ and $\psi_{\mathrm{SR}}^{\star}$ abbreviated to $p^{\star}$ and $\psi^{\star}$, introduce the curve

$$
S_{\mathrm{PS}, t}(\mathrm{p})= \begin{cases}-k_{\mathrm{Co}} & \text { for } \mathrm{p}<\psi^{\star}(t) \\ {\left[-k_{\mathrm{Co}}, k_{\mathrm{Co}}\right]} & \text { for } \mathrm{p}=\psi^{\star}(t) \\ k_{\mathrm{Co}} & \text { for } \mathrm{p}>\psi^{\star}(t)\end{cases}
$$

and add it to the $S_{\mathrm{Th}}$ of (2.1) to form

$$
S_{t}(\mathrm{p})=S_{\mathrm{Th}}(\mathrm{p})+S_{\mathrm{PS}, t}(\mathrm{p}) .
$$

For every $t$, this curve intersects $D_{t}$ at $p^{\star}(t)$. When $p^{\star}(t)=\psi^{\star}(t)$, the rate of equilibrium output from storage can be read off as the horizontal distance from the intersection point to the centre of the horizontal segment of length $2 k_{\text {Co }}$ which $S_{t}$ has at the price $\psi^{\star}(t)$ : see Figure 2. As the point is, respectively, left or right of centre, so the output is negative or positive, i.e., the reservoir is being charged or discharged.

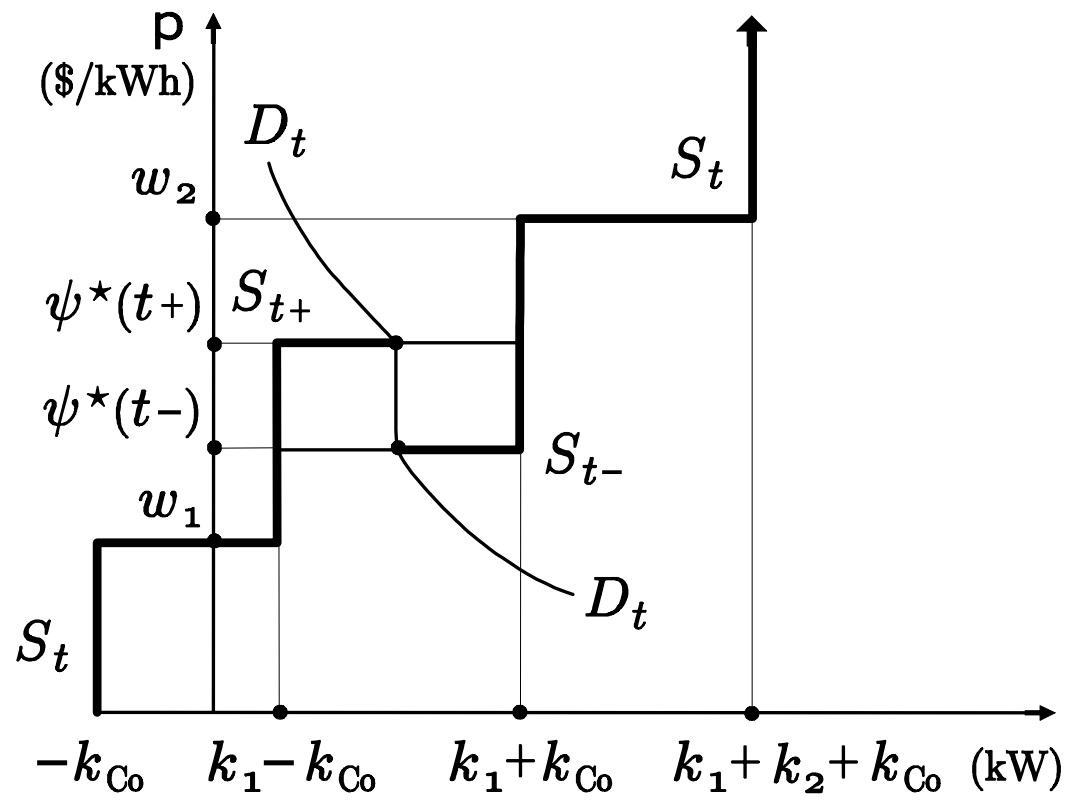

Figure 2. Proof of continuity of short-run equilibrium price for electricity supplied by a combined generation-and-storage system: the energy stock price $\psi^{\star}$ cannot be discontinuous if the (cross-price independent) demand curve $D_{t}$ is strictly decreasing; and if $\psi^{\star}(t)$ is continuous in $t$, then so is the supply curve $S_{t}$ and hence also $p^{\star}(t)$.

Suppose that $\psi^{\star}$ has a jump at some $t$, i.e., $\psi^{\star}(t-)<\psi^{\star}(t+)$. Say there is no $w_{\theta}$ between $\psi^{\star}(t-)$ and $\psi^{\star}(t+)$. (If there is, it only helps the argument.) Figure 2 shows the case of $\Theta=2$ with $w_{1}<\psi^{\star}(t-)<\psi^{\star}(t+)<w_{2}$; the curve given by (2.1) plus (3.1) with $\psi^{\star}(t-)$ or $\psi^{\star}(t+)$ in place of $\psi^{\star}(t)$ is denoted by $S_{t-}$ or $S_{t+}$. Now note that $D_{t}$ cannot intersect $S_{t+}$ below $\psi^{\star}(t+)$ or to the left of centre of the 
horizontal segment at the level $\psi^{\star}(t+)$, since this would mean that the reservoir is being charged for a time just after $t$, which is infeasible because the reservoir is full at $t .{ }^{10}$ Similarly, $D_{t}$ cannot intersect $S_{t-}$ above $\psi^{\star}(t-)$ or to the right of centre of the horizontal segment at the level $\psi^{\star}(t-)$, since this would mean that the reservoir is being discharged for a time just before $t$, which is again infeasible. (In Figure 2, the lines that $D_{t}$ cannot intersect are the heavy lines.) So, being monotone, $D_{t}$ must have a vertical segment, from the centre at level $\psi^{\star}(t+)$ to the centre at level $\psi^{\star}(t-)$. But such a vertical segment contradicts the strict monotonicity of $D_{t}$ in p, i.e., the differentiability of $u(t, \cdot)$. This shows that $\psi^{\star}$ is continuous, and hence so is $p^{\star}$.

In Section 9 , the continuity of $p^{\star}$ and $\hat{\psi}\left(p^{\star}\right)$ is re-derived in a different way, and in the other order: first it is proved for $p^{\star}$ by applying Theorem 7.2. And if $p$ is continuous then so is $\hat{\psi}(p)$ - see $[11] .^{11}$

\section{Commodity And PRICE SPACEs For A General Framework}

The method of curves is limited to the case of independent demands (Sections 2 and 3). A more general price continuity result can be based on the same idea, viz., that a price jump would cause oppositely signed discontinuities in the supply and demand trajectories. The resulting demand drop must be nonzero to include the case when supply does not actually jump but does not drop either (as in Figure 1a, where $\mathrm{y}$ stays at $k_{1}$ if $\mathrm{p}$ jumps from $w_{1}$ to $w_{2}$ ). For a general result (Theorem 7.2), such properties are simply assumed. We name them sub-symmetry and quasi-symmetry of preferences and technologies, since these properties follow from the stronger condition of symmetry, i.e., invariance under rearrangement. A function $C$ of $(y(t))_{t \in[0, T]}$ is symmetric (a.k.a. rearrangement-invariant) if $C(y)$ depends only on the distribution of $y$ w.r.t. the Lebesgue measure on $[0, T]{ }^{12}$ For example, the SR cost of thermal electricity generation, $C_{\mathrm{SR}}$ of $(2.2)$, is symmetric. (So is the LR cost, which is not additively separable like $C_{\mathrm{SR}}$.) When $C$ is convex, its symmetry ensures that $y$ and $p=\nabla C(y)$ are similarly arranged, i.e., that for (almost) every $t^{\prime}$ and $t^{\prime \prime}$ if $p\left(t^{\prime}\right)<p\left(t^{\prime \prime}\right)$ then $y\left(t^{\prime}\right) \leq y\left(t^{\prime \prime}\right)$ : see $[9$, Theorem 1].

Applied to a joint cost as a function of the output trajectory $y$, this means that outputs are always higher (or at least not lower) at higher-priced times. In other words, price and output increments do not have opposite signs anywhere on $[0, T]$. This is a global price-output relationship; and its full strength is not necessary in proving price continuity, which is a local property of the equilibrium price function $p^{\star}:[0, T] \rightarrow \mathbb{R}$. A local and approximate relationship between the quantities and the supporting prices suffices. This is sub-symmetry (Definition 5.11). On the production side the assumption is further weakened to quasi-symmetry (Definition 5.14), to

\footnotetext{
${ }^{10}$ Continuity of $D_{t}$ over $t$ is used here.

${ }^{11}$ Obviously this cannot be used to derive the continuity of $p^{\star}$, as in this section, from the continuity of $\psi^{\star}$.

${ }^{12}$ Equivalently, $C$ is symmetric if $C(y)=C(y \circ \rho)$ for every Lebesgue measure-preserving transformation $\rho:[0, T] \rightarrow[0, T]$.
} 
make it hold for energy storage (Lemma 9.1). On the consumption side the assumption is slightly strengthened (Definition 6.1). It is verified for additively separable utility (Example 6.2). Given Lemma 9.1, this re-establishes the result of Section 3. Extensions to other forms of utility are sketched (Example 6.3).

Such an analysis requires the framework of a dual pair of commodity and price spaces. In peak-load pricing an output bundle is always bounded by productive capacity, so the commodity space is $L^{\infty}[0, T]$. It is paired with $L^{1}[0, T]$ as the price space; but the task is to show that the equilibrium price density $p^{\star}$ is continuous (and not just integrable) on $[0, T]$. Before this application, $T$ denotes an abstract set of commodities which carries a topology with a countable base of open sets. Additionally $T$ carries a finite nonatomic (and nonnegative) measure $\sigma$ on a sigma-algebra $\mathcal{A}$ that contains all the Borel subsets of $T$. Every nonempty open subset of $T$ is assumed to be $\sigma$-nonnull, i.e., to have a positive measure. The vector space of all ( $\sigma$-equivalence classes of ) $\mathcal{A}$-measurable real-valued functions on $T$ is denoted by $L^{0}(T, \sigma)$. The space of all $\sigma$-essentially bounded functions, $L^{\infty}(T)$, is paired with $L^{1}(T)$, the space of all $\sigma$-integrable functions. (The analysis can be adapted for use with other $L^{\rho}$-spaces in problems involving unbounded commodity bundles.)

Apart from $T$ (which may represent a single differentiated good), there is a finite number of homogeneous goods numbered by $1,2, \ldots, G \geq 0$. So a complete commodity bundle is an $(x, m) \in L^{\infty}(T) \times \mathbb{R}^{G}$, and its value at a price system $(p, r) \in L^{1}(T) \times \mathbb{R}^{G}$ is $\int_{T} p(t) x(t) \sigma(\mathrm{d} t)+r \cdot m$, abbreviated to $\langle p, x\rangle+r \cdot m$.

\section{Symmetry AND WEAKER CONDitions ON PRODUCTION SETS}

The idea that a jump in the price trajectory cannot coincide with a drop in the supply trajectory is next developed. First this is shown to follow from the symmetry of the cost function (or of the input correspondence when the inputs are not aggregated into a scalar cost). Actually symmetry implies a stronger "similarity" between the price and output trajectories, viz., that they rise and fall simultaneously (Lemma 5.9). This is more than is needed for price continuity, but the assumption is too strong for some applications: in electricity pricing the storage cost is not symmetric (although the thermal cost is). The similarity condition is therefore weakened (Definitions 5.11, 5.14 and 5.17). Like symmetry, the weaker properties are preserved in the summation of production sets. Some "general" examples meeting the weak conditions are given at this stage, but the motivating example of energy storage is studied in Section 9 (Lemmas 9.1 and 9.3).

Definition 5.1. A function $C$ on $L^{0}(T)$ is $\sigma$-symmetric (a.k.a. rearrangement-invariant) if its value depends only on the distribution of its argument, w.r.t. $\sigma$. (In other words, $C$ is symmetric if the condition $\sigma\left(y^{-1}(B)\right)=\sigma\left(z^{-1}(B)\right)$ for every Borel set $B \subset \mathbb{R}$ implies that $C(y)=C(z)$.)

Definition 5.2. A set $S \subset L^{0}(T)$ is $\sigma$-symmetric if its indicator function is symmetric, i.e., if $z \in S$ whenever $z$ has the same distribution, w.r.t. $\sigma$, as some 
$y \in S$. (In other words, $S$ is symmetric if the conditions: $y \in S, z \in L^{0}$ and $\sigma\left(y^{-1}(B)\right)=\sigma\left(z^{-1}(B)\right)$ for every Borel set $B \subset \mathbb{R}$ imply that $z \in S$ also.)

This concept is used with $S$ equal to the section of a production set $\mathbb{Y} \subset L^{\infty}(T) \times \mathbb{R}^{G}$ through a $q \in \mathbb{R}^{G}$, i.e., with $S$ equal to the $q$-restricted production set

$$
\mathbb{Y}(q):=\left\{y \in L^{\infty}(T):(y, q) \in \mathbb{Y}\right\} .
$$

The set of "output" bundles $\mathbb{Y}(q)$ is symmetric for each "input" $q \in \mathbb{R}^{G}$ if and only if the "input requirement" set $\mathbb{Y}_{y}:=\{q:(y, q) \in \mathbb{Y}\}$ depends only on the distribution of $y$ (w.r.t. $\sigma$ ). In such a case the cost

$$
C(y):=\inf _{q}\{-\langle r, q\rangle:(y, q) \in \mathbb{Y}\}
$$

is a symmetric function of $y$ (for each input price system $r \in \mathbb{R}^{G}$ ).

For the purpose of proving price continuity on $T$, the relevant implication of symmetry is the similarity of arrangement for the functions $p$ and $y$ (on $T$ ) which represent, respectively, a price system and an output bundle that maximises the (q-restricted) profit on $S=\mathbb{Y}(q)$. This result (Lemma 5.9) is preceded by a discussion of the similarity concept, introduced (for functions) by Day [3, p. 932].

Definition 5.3. Two elements, $p$ and $y$, of $L^{0}(T)$ are similarly arranged if, for any measurable sets $A^{\prime}$ and $A^{\prime \prime},{ }^{13}$

$$
\operatorname{ess} \sup _{A^{\prime}} p<\operatorname{ess} \inf _{A^{\prime \prime}} p \Rightarrow \operatorname{ess} \sup _{A^{\prime}} y \leq \operatorname{ess} \inf _{A^{\prime \prime}} y .
$$

With $p$ and $y$ replaced by any of their variants $\breve{p}$ and $\breve{y}-$ which are literally functions rather than classes of almost everywhere (a.e.) equal functions - similarity of arrangement can also be defined in terms of values at any points $t^{\prime}$ and $t^{\prime \prime}$ (instead of values on nonnull sets $A^{\prime}$ and $A^{\prime \prime}$ ).

Remark 5.4. Two elements, $p$ and $y$, of $L^{0}(T)$ are similarly arranged if and only if

$$
p\left(t^{\prime}\right)<p\left(t^{\prime \prime}\right) \Rightarrow y\left(t^{\prime}\right) \leq y\left(t^{\prime \prime}\right)
$$

for $\sigma$-almost every (a.e.) $t^{\prime}$ and $t^{\prime \prime}$ in $T$-i.e., if and only if for any variants $\breve{p}$ and $\breve{y}$ (of $p$ and $y$ ) there is a $\sigma$-null set $Z$ such that for every $t^{\prime}$ and $t^{\prime \prime}$ in $T \backslash Z$

$$
\breve{p}\left(t^{\prime}\right)<\breve{p}\left(t^{\prime \prime}\right) \Rightarrow \breve{y}\left(t^{\prime}\right) \leq \breve{y}\left(t^{\prime \prime}\right) .
$$

Proof. To show that (5.3) implies (5.2), take any measurable sets $A^{\prime}$ and $A^{\prime \prime}$ satisfying the antecedent of (5.2). There exist null sets $Z^{\prime}$ and $Z^{\prime \prime}$ (which generally depend on $A^{\prime}$ and $A^{\prime \prime}$ ) such that

$$
\sup _{A^{\prime} \backslash Z^{\prime}} \breve{p}=\operatorname{ess} \sup _{A^{\prime}} p<\operatorname{ess} \inf _{A^{\prime \prime}} p=\inf _{A^{\prime \prime} \backslash Z^{\prime \prime}} \breve{p} .
$$

\footnotetext{
${ }^{13}$ It obviously suffices to verify this for any $\sigma$-almost disjoint pair of $\sigma$-nonnull sets, $A^{\prime}$ and $A^{\prime \prime}$. (If $\sigma(A)=0$ then $\operatorname{ess}_{\sup _{A}} y=-\infty$ and essinf $\operatorname{in}_{A} y=+\infty$. So, in Definition 5.3, if $A^{\prime}$ or $A^{\prime \prime}$ is null then the consequent holds, for any $y$. And if $\sigma\left(A^{\prime} \cap A^{\prime \prime}\right)>0$ then the antecedent fails, for any $p$.)
} 
One can assume that $Z^{\prime} \supseteq Z$ and $Z^{\prime \prime} \supseteq Z$ (by replacing $Z^{\prime}$ and $Z^{\prime \prime}$ with $Z^{\prime} \cup Z$ and $\left.Z^{\prime \prime} \cup Z\right) .{ }^{14}$ By (5.3), applied to every $t^{\prime} \in A^{\prime} \backslash Z^{\prime}$ and $t^{\prime \prime} \in A^{\prime \prime} \backslash Z^{\prime \prime}$, it follows that

$$
\text { ess } \sup _{A^{\prime}} y \leq \sup _{A^{\prime} \backslash Z^{\prime}} \breve{y} \leq \inf _{A^{\prime \prime} \backslash Z^{\prime \prime}} \breve{y} \leq \operatorname{ess} \inf _{A^{\prime \prime}} y
$$

as required.

To show that, conversely, (5.2) implies (5.3), fix any variants $\breve{p}$ and $\breve{y}$, and let $Z$ be a null set (to be specified later). Given any $t^{\prime}$ and $t^{\prime \prime}$ in $T \backslash Z$ with $\breve{p}\left(t^{\prime}\right)<\breve{p}\left(t^{\prime \prime}\right)$, take any $\underline{\varrho}$ and $\bar{\varrho}$ from $\mathbb{Q}$ (the set of all rational numbers) such that $\breve{p}\left(t^{\prime}\right)<\underline{\varrho}<\bar{\varrho}<\breve{p}\left(t^{\prime \prime}\right)$, and $\overline{\text { define }}$

$$
\begin{aligned}
& A^{\prime}:=\{t \in T: \breve{p}(t)<\underline{\varrho}\} \\
& A^{\prime \prime}:=\{t \in T: \bar{\varrho}<\breve{p}(t)\} .
\end{aligned}
$$

Since

$$
\operatorname{ess} \sup _{A^{\prime}} p \leq \underline{\varrho}<\bar{\varrho} \leq \operatorname{ess} \inf _{A^{\prime \prime}} p
$$

it follows from (5.2) that

$$
\text { ess } \sup _{A^{\prime}} y \leq \operatorname{ess} \inf _{A^{\prime \prime}} y \text {. }
$$

Once $Z$ is chosen to ensure that

$$
\sup _{A^{\prime} \backslash Z} \breve{y}=\operatorname{ess} \sup _{A^{\prime}} y \quad \text { and } \quad \operatorname{ess} \inf _{A^{\prime \prime}} y=\inf _{A^{\prime \prime} \backslash Z} \breve{y}
$$

it will follow (since $t^{\prime} \in A^{\prime} \backslash Z$ and $t^{\prime \prime} \in A^{\prime \prime} \backslash Z$ ) that

$$
\breve{y}\left(t^{\prime}\right) \leq \sup _{A^{\prime} \backslash Z} \breve{y} \leq \inf _{A^{\prime \prime} \backslash Z} \breve{y} \leq \breve{y}\left(t^{\prime \prime}\right)
$$

as required. To complete the argument, for each $\varrho \in \mathbb{Q}$ define $A_{\varrho}^{\prime}$ and $A_{\varrho}^{\prime \prime}$ as in (5.4) and (5.5) but with $\varrho$ instead of the $\varrho$ or $\bar{\varrho}$, and take two null sets $Z_{\varrho}^{\prime}$ and $Z_{\varrho}^{\prime \prime}$ such that: (i) the (ordinary) supremum of $\breve{y}$ on $A_{\varrho}^{\prime} \backslash Z_{\varrho}^{\prime}$ equals the essential supremum of $y$ on $A_{\varrho}^{\prime}$, and (ii) the (ordinary) infimum of $\breve{y}$ on $A_{\varrho}^{\prime \prime} \backslash Z_{\varrho}^{\prime \prime}$ equals the essential infimum of $y$ on $A_{\varrho}^{\prime \prime}$. Define

$$
Z:=\bigcup_{\varrho \in \mathbb{Q}}\left(Z_{\varrho}^{\prime} \cup Z_{\varrho}^{\prime \prime}\right)
$$

This set is a null set that depends on $\breve{p}$ and $\breve{y}$; and it has the required property (5.6) because $A^{\prime}=A_{\underline{\varrho}}^{\prime}$ and $A^{\prime \prime}=A_{\underline{\varrho}}^{\prime \prime} \cdot{ }^{15}$

\footnotetext{
${ }^{14}$ One can also choose $Z^{\prime}$ to ensure that the essential range of $p$ on $A^{\prime}$ equals cl $\breve{p}\left(A^{\prime} \backslash Z^{\prime}\right)$, etc.

${ }^{15}$ This proof shows indirectly, by using $A^{\prime} \backslash Z \neq \emptyset \neq A^{\prime \prime} \backslash Z$ to establish (5.6) and (5.7), that both $A^{\prime}$ and $A^{\prime \prime}$ are nonnull. This can also be seen directly: for any $\varrho$, if (say) $A_{\varrho}^{\prime}$ is null then $Z_{\varrho}^{\prime}$ must equal $A_{\varrho}^{\prime}$. In other words, removing the set $Z$ removes all the null sets of the form $\{t: \breve{p}(t)<\varrho\}$ or $\{t: \varrho<\breve{p}(t)\}$, with a rational $\varrho$. So the essential extrema of $p$ (on $T$ ) are equal to the ordinary extrema of $\breve{p}$ on $T \backslash Z$. For comparison, if all the null sets of the form $\left\{t: \varrho_{1}<\breve{p}(t)<\varrho_{2}\right\}$, with
} 
Similarity of arrangement is equivalent to the existence of a common ranking pattern, as is shown by Day [3, p. 939, 5.6]. To state this, we first introduce the concepts.

Definition 5.5. The nonincreasing rearrangement $y_{\downarrow}$ of a $y \in L^{0}(T, \sigma)$ is the nonincreasing function on $[0, \sigma(T)]$ with the same distribution, relative to meas (the Lebesgue measure), as that of $y$ w.r.t. $\sigma .{ }^{16}$

Definition 5.6. A ranking pattern of a $y \in L^{0}(T, \sigma)$ is any measure-preserving map $\rho: T \rightarrow[0, \sigma(T)]$ such that $y=y_{\downarrow} \circ \rho$; the set of all such maps is denoted by $\mathcal{R}(y) .{ }^{17}$

Comments:

1. That $\mathcal{R}(y) \neq \emptyset$ (if $\sigma$ is nonatomic) is the Lorentz-Ryff Lemma [24, Lemma 1], stated also in, e.g., $[4,3.3]$.

2. If $y$ has no plateau (i.e., $\sigma\{t: y(t)=\mathrm{y}\}=0$ for each $\mathrm{y} \in \mathbb{R}$ or, equivalently, $y_{\downarrow}$ is strictly decreasing), then the pattern of $y$ is unique, and it is $\rho_{y}=\left(y_{\downarrow}\right)^{-1} \circ y$. Note that

$$
\rho_{y}(t)=\sigma\{\tau \in T: y(\tau) \geq y(t)\}
$$

i.e., $\rho_{y}(t) / \sigma(T)$ is $t$ 's "percentage above" (the fraction of $T$ on which $y$ is above $y(t))$. Thus $\rho_{y}$ ranks the elements of $T$ by the value of $y$.

Remark 5.7 (Day). Assume that $\sigma$ is nonatomic (on $\mathcal{A}$ ). Two functions $p$ and $y$, in $L^{0}(T, \mathcal{A}, \sigma)$, are similarly arranged if and only if

$$
\mathcal{R}(p) \cap \mathcal{R}(y) \neq \emptyset
$$

i.e., if and only if both $p=p_{\downarrow} \circ \rho$ and $y=y_{\downarrow} \circ \rho$ for some measure-preserving map $\rho: T \rightarrow[0, \sigma(T)]$.

\section{Comments:}

1. It is obvious that (5.8) implies (5.3) and, equivalently, (5.2). To prove the converse - that (5.2) implies (5.8)-Day [3, p. 939, 5.6] extends the LorentzRyff Lemma to pairs (and $n$-tuples) of functions, and adds an argument which yields another equivalent condition.

2. In (5.2) and (5.3), the inequalities in the antecedent and the consequent must be strict and nonstrict, respectively.

3. As is obvious from Remark 5.4 (or Remark 5.7), similarity of arrangement is a symmetric binary relation (in $L^{0}$ ) -i.e., $p$ and $y$ can be interchanged in (5.2) or (5.3). ${ }^{18}$ It is not a transitive relation (since every function is arranged similarly to a constant).

rational $\varrho_{1}$ and $\varrho_{2}$, were removed from $T$, then the range of $\breve{p}$ on the remainder of $T$ would be dense in the essential range of $p$ on $T$ (defined as the smallest closed set $E \subseteq \mathbb{R}$ with $\sigma\{t: p(t) \notin E\}=0$ ).

${ }^{16}$ When $y$ is the output and $p$ is a TOU tariff, the graphs of $y_{\downarrow}$ and $p_{\downarrow}$ are known in electricity pricing as the load- and price-duration curves. On $L^{\infty}$, the operation $x \mapsto x_{\downarrow}$ is $\mathrm{m}\left(L^{\infty}, L^{1}\right)$ continuous: see [7].

17 "Measure-preserving" means that $\sigma\left(\rho^{-1}(B)\right)=$ meas $B$ for every Borel set $B \subset[0, \sigma(T)]$.

${ }^{18}$ This can also be shown directly from Definition 5.3 . 
Similarity of arrangement is preserved in summation.

Remark 5.8. If each of two functions, $y$ and $z$, is arranged similarly to $p$, then so is $y+z$.

Proof. This follows from, e.g., Definition 5.3 and the inequalities

$$
\begin{aligned}
\operatorname{ess} \sup _{A^{\prime}}(y+z) & \leq \operatorname{ess} \sup _{A^{\prime}} y+\operatorname{ess} \sup _{A^{\prime}} z \\
\text { ess } \inf _{A^{\prime \prime}} y+\operatorname{ess} \inf _{A^{\prime \prime}} z & \leq \operatorname{ess} \inf _{A^{\prime \prime}}(y+z)
\end{aligned}
$$

for every $y$ and $z$.

As has been mentioned, if $p$ represents a linear functional supporting a symmetric set $S$ at a point $y$, then $p$ and $y$ are similarly arranged (or, equivalently, have a common pattern). This is next spelt out for the case of $p \in L^{1}$ and $y \in S \subset L^{\infty}$. (The same holds for $L^{\rho}$ and $L^{\rho^{\prime}}$ instead of $L^{\infty}$ and $L^{1}$.)

Lemma 5.9. Assume that the measure $\sigma$ is nonatomic (on $\mathcal{A}$ ) and that $S$ is a symmetric subset of $L^{\infty}(T, \mathcal{A}, \sigma)$. If $p \in L^{1}(T)$ and $y$ maximises $\langle p, \cdot\rangle$ on $S-i . e ., y \in S$ and $\langle p, y\rangle=\sup \{\langle p, z\rangle: z \in S\}$-then $p$ and $y$ are similarly arranged.

Proof. It suffices to show that $\langle p, y\rangle \geq\left\langle p_{\downarrow}, y_{\downarrow}\right\rangle$, which is the reverse of the HardyLittlewood-Pólya Inequality, and then to apply Day's characterisation of the case of equality $\left[3,5.2\right.$, pp. 937-938]. ${ }^{19}$ To this end, since $\sigma$ is nonatomic, take any $\rho \in \mathcal{R}_{p}$ (i.e., $p=p_{\downarrow} \circ \rho$ ). Since $S$ is symmetric, $y_{\downarrow} \circ \rho \in S$; so

$$
\langle p, y\rangle \geq\left\langle p, y_{\downarrow} \circ \rho\right\rangle=\left\langle p_{\downarrow} \circ \rho, y_{\downarrow} \circ \rho\right\rangle=\left\langle p_{\downarrow}, y_{\downarrow}\right\rangle
$$

(since $\rho$ is measure-preserving). So $\langle p, y\rangle=\left\langle p_{\downarrow}, y_{\downarrow}\right\rangle$, and Day's result shows that $\mathcal{R}(p) \cap \mathcal{R}(y) \neq \emptyset$.

Comment: In the Proof of Lemma 5.9, $\rho$ is a pattern of $p$ but not of $y$, in general. If, however, $p$ has no plateau, then it has a single pattern, and so $\rho$ must be the common pattern of $p$ and $y$.

The corresponding result for functions follows [9, Theorem 1].

Remark 5.10. If $\sigma$ is nonatomic, $C: L^{\infty}(T, \sigma) \rightarrow \mathbb{R}$ is a symmetric convex function and $p \in \partial C(y) \cap L^{1}(T, \sigma)$, i.e., a $p \in L^{1}$ is a subgradient of $C$ at $y$, then $p$ and $y$ are similarly arranged.

Applied to a (restricted) production set $S=\mathbb{Y}(q)$, Lemma 5.9 shows that, in an output bundle $y \in S \subset L^{\infty}(T)$ and a supporting price system $p \in L^{1}(T)$, the quantity and price move up and down together over "time". For the purpose of proving price continuity, a weaker property suffices.

Notation: The set of all neighbourhoods of $t$ is denoted by $\mathcal{N}(t)$.

\footnotetext{
${ }^{19}$ The inequality is: $\langle p, y\rangle \leq\left\langle p_{\downarrow}, y_{\downarrow}\right\rangle$, with equality if and only if $p$ and $y$ are similarly arranged. This is a special case of $[3,5.2]$, for $\varphi\left(x_{1}, x_{2}\right)=x_{1} x_{2}$.
} 
Definition 5.11. A set $S \subset L^{\infty}(T)$ is sub-symmetric if:

For every $p \in L^{1}(T)$ and every $y$ that maximises $\langle p, \cdot\rangle$ on $S$, and for every $t \in T$ and $\epsilon>0$, there exists an $H \in \mathcal{N}(t)$ such that for any two measurable sets $A^{\prime} \subset H$ and $A^{\prime \prime} \subset H$

$$
\epsilon+\operatorname{ess} \sup _{A^{\prime}} p<\operatorname{ess} \inf _{A^{\prime \prime}} p \Rightarrow \operatorname{ess} \sup _{A^{\prime}} y \leq \operatorname{ess} \inf _{A^{\prime \prime}} y \text {. }
$$

Equivalently ${ }^{20}$ for every $p, y, t$ and $\epsilon$ such as above, there exists an $H \in \mathcal{N}(t)$ such that, for $\sigma$-almost every (a.e.) $t^{\prime}$ and $t^{\prime \prime}$ in $H$,

$$
\epsilon+p\left(t^{\prime}\right)<p\left(t^{\prime \prime}\right) \Rightarrow y\left(t^{\prime}\right) \leq y\left(t^{\prime \prime}\right) \text {. }
$$

\section{Comments:}

1. The $p$ and $y$ in (5.12) must of course be interpreted as (any) variants $\breve{p}$ and $\breve{y}$, as in (5.3). The phrase "for a.e. $t^{\prime}$ and $t^{\prime \prime}$ in $H$ " is also to be interpreted as in Remark 5.4-viz., as meaning "for every $t^{\prime}$ and $t^{\prime \prime}$ in $H$ but outside of some $\sigma$-null set $Z$ ". The excepted set $Z$ depends on the choice of variants $\breve{p}$ and $\breve{y}$ (but it can be chosen independently of $\epsilon$ ).

2. Unlike the case of a symmetric $S$ (in which $p$ and $y$ are similarly arranged by Lemma 5.9), with a sub-symmetric $S$ the relationship between $p$ and $y$ is not symmetric - i.e., $p$ and $y$ cannot be interchanged in (5.11) or (5.12).

3 . Because of the $\epsilon$, the strict inequality between the values of $p$ in the antecedent of (5.11) or (5.12) can be made nonstrict without changing the concept. But the inequality between the values of $y$ in the consequent of (5.11) or (5.12) must be non-strict, as in (5.2) or (5.3).

4. Every symmetric set is sub-symmetric: by Lemma 5.9 (and Definition 5.3), to satisfy (5.11) it suffices to set $H=T$ (regardless of $\epsilon$ ).

A proper example of sub-symmetry in production is the additively separable convex cost function $\int_{T} c(t, y(t)) \mathrm{d} t$ : it is not symmetric unless the "instantaneous" cost is independent of $t$ directly (i.e., unless the integrand $c(t, \mathrm{y})$ is actually independent of $t$, as in (2.2)). But if the cost curve $c(t, \cdot)$, together with its $\mathbf{y}$-derivative, varies continuously with $t$, then it can be approximated in a neighbourhood of any $t_{0} \in T$ by the fixed (time-independent) curve $c\left(t_{0}, \cdot\right)$. This is why $\int c(t, y(t)) \mathrm{d} t$ is "locally and approximately" symmetric - i.e., sub-symmetric.

Example 5.12. Assume that $c: T \times(-\infty, \mathrm{k}] \rightarrow \mathbb{R}$, where $\mathrm{k} \in \mathbb{R}$ is a constant, is a differentiable convex integrand, i.e., the function $t \mapsto c(t, \mathrm{y})$ is $\sigma$-integrable on $T$ (for every $\mathrm{y} \in \mathbb{R})$, and that the function $\mathrm{y} \mapsto c(t, \mathrm{y})$ is convex and differentiable on $(-\infty, \mathrm{k}]$, for every $t \in T$. Then

$$
C(y):=\int_{T} c(t, y(t)) \sigma(\mathrm{d} t)
$$

is a convex integral functional on $L^{\infty}(T)$, defined effectively for $y \leq \mathrm{k}$ : see, e.g., [22].

\footnotetext{
${ }^{20}$ The equivalence of $(5.11)$ to $(5.12)$ can be proved in the same way as Remark 5.4.
} 
If additionally $\partial c / \partial \mathbf{y}$ is (jointly) continuous on $T \times(-\infty, \mathbf{k}]$ then, for any $m \in \mathbb{R}$, the set

$$
S=\left\{y \in L^{\infty}(T): C(y) \leq m\right\}
$$

is sub-symmetric, provided that $C(y)<m$ for some $y$ (Slater's Condition).

Comments:

1. $(\partial c / \partial \mathbf{y})(t, \mathbf{k})$ means the left (one-sided) derivative (w.r.t. $\mathbf{y}$, at $\mathbf{y}=\mathbf{k}$ ), which is assumed to be finite. If there were no capacity constraint $\mathbf{k}$, then $c(t, \cdot)$ might be defined on $\mathbb{R}$ instead of $(-\infty, k]$. Example 5.12 extends to that case.

2. With $c$ differentiable in $\mathrm{y}$ on $(-\infty, \mathbf{k})$, a strict inequality holds between the values of $y$ in (5.12), except when $y\left(t^{\prime}\right)=y\left(t^{\prime \prime}\right)=\mathrm{k}$. The exception is caused by the kink which $c(t, \cdot)$ has at $\mathrm{y}=\mathrm{k}$ (where the curve is "cut off" by setting $c(t, \mathrm{y})=+\infty$ for $\mathbf{y}>\mathbf{k})$ : see (5.16).

3 . When a negative $y(t)$ can arise only from free disposal, the "instantaneous" production cost $c(t, \mathrm{y})$ is nondecreasing in $\mathrm{y}$, with $c(t, \mathrm{y})=0$ for $\mathrm{y} \leq 0$. And $c(t, \cdot)$ can have a kink at $\mathrm{y}=0$ without spoiling the result (just as it has a kink at $\mathbf{y}=\mathbf{k})$, i.e., Example 5.12 extends to the case of $(\partial c / \partial \mathrm{y})(t, 0+)>0$.

4. For (5.12) to hold as it is, the $y$-coordinate of any kinks in $c(t, \cdot)$ must be independent of $t$, as in the above cases of $\mathrm{y}=\mathrm{k}$ or 0 . If the inequality $y\left(t^{\prime}\right) \leq$ $y\left(t^{\prime \prime}\right)$ in (5.12) were relaxed to $y\left(t^{\prime}\right) \leq y\left(t^{\prime \prime}\right)+\epsilon$, then Example 5.12 could be generalised to the case of any convex $c(t, \cdot)$ with a subdifferential $\partial_{\mathrm{y}} c(t, \mathrm{y})$ that is continuous in $t$ (as a correspondence).

Proof of Example 5.12. Take any $t \in T, \epsilon>0, p \in L^{1}(T)$ and $y$ that maximises $\langle p, \cdot\rangle$ on $S$. There exists a scalar $\mu \geq 0$ such that ${ }^{21}$

$$
\begin{aligned}
& p(\tau)=\mu \frac{\partial c}{\partial \mathrm{y}}(\tau, y(\tau)) \quad \text { for } \sigma \text {-almost every } \tau \in T \text { with } y(\tau)<\mathrm{k} \\
& p(\tau) \geq \mu \frac{\partial c}{\partial \mathrm{y}}(\tau, \mathrm{k}) \quad \text { for } \sigma \text {-almost every } \tau \in T \text { with } y(\tau)=\mathrm{k} .
\end{aligned}
$$

If $\mu>0$ then, by Corollary B.2 applied to $M=-\mu \partial c / \partial y,{ }^{22}$ there exists an $H \in \mathcal{N}(t)$ such that: for every $t^{\prime}$ and $t^{\prime \prime}$ in $H$ and every $\mathrm{y}^{\prime}$ and $\mathrm{y}^{\prime \prime}$ in $[\operatorname{EssInf}(y), \operatorname{EssSup}(y)] \subset$ $(-\infty, k]$

$$
\mathrm{y}^{\prime}>\mathrm{y}^{\prime \prime} \Rightarrow \frac{\partial c}{\partial \mathrm{y}}\left(t^{\prime}, \mathrm{y}^{\prime}\right) \geq \frac{\partial c}{\partial \mathrm{y}}\left(t^{\prime}, \mathrm{y}^{\prime \prime}\right) \geq \frac{\partial c}{\partial \mathrm{y}}\left(t^{\prime \prime}, \mathrm{y}^{\prime \prime}\right)-\frac{\epsilon}{\mu}
$$

From this and (5.15)-(5.16), for a.e. $t^{\prime}$ and $t^{\prime \prime}$ in $H$,

$$
y\left(t^{\prime}\right)>y\left(t^{\prime \prime}\right) \Rightarrow p\left(t^{\prime}\right) \geq \mu \frac{\partial c}{\partial y}\left(t^{\prime}, y\left(t^{\prime}\right)\right) \geq \mu \frac{\partial c}{\partial y}\left(t^{\prime \prime}, y\left(t^{\prime \prime}\right)\right)-\epsilon=p\left(t^{\prime \prime}\right)-\epsilon .
$$

\footnotetext{
${ }^{21}$ This is where Slater's Condition is used.

${ }^{22}$ Here it suffices to use the partial continuity of $\partial c / \partial \mathrm{y}$ in $t$, which holds uniformly in $\mathrm{y}$.
} 
In other words, for a.e. $t^{\prime}$ and $t^{\prime \prime}$ in $H$,

$$
\epsilon+p\left(t^{\prime}\right)<p\left(t^{\prime \prime}\right) \Rightarrow y\left(t^{\prime}\right) \leq y\left(t^{\prime \prime}\right)
$$

which proves (5.12).

Finally, if $\mu=0$ then $p\left(t^{\prime \prime}\right)>p\left(t^{\prime}\right)$ implies that $y\left(t^{\prime \prime}\right)=\mathbf{k} \geq y\left(t^{\prime}\right)$, as required.

This example is next reoriented for application to an industrial customer using a differentiated input $z=-y \in L_{+}^{\infty}(T)$ to produce a quantity $\int_{T} f(t, z(t)) \mathrm{d} t$ of a homogeneous output good.

Example 5.13. Assume that $f: T \times \mathbb{R}_{+} \rightarrow \mathbb{R}$ is a differentiable concave integrand, i.e., the function $t \mapsto f(t, \mathbf{z})$ is $\sigma$-integrable on $T$ (for every $\mathbf{z} \in \mathbb{R}$ ), whilst the function $\mathbf{z} \mapsto f(t, \mathbf{z})$ is concave and differentiable on $\mathbb{R}_{+} \cdot{ }^{23}$ Then

$$
F(z):=\int_{T} f(t, z(t)) \sigma(\mathrm{d} t)
$$

is a concave integral functional on $L_{+}^{\infty}(T)$ : see, e.g., [22].

If additionally $\partial f / \partial \mathbf{z}$ is (jointly) continuous on $T \times \mathbb{R}_{+}$then, for any $\varphi \in \mathbb{R}$, the set

$$
S=\left\{-z \in L_{-}^{\infty}(T): F(z) \geq \varphi\right\}
$$

is sub-symmetric, provided that $F(z)>\varphi$ for some $z$ (Slater's Condition).

Comment: With $f$ taken to be differentiable in $\mathrm{z}$ on $\mathbb{R}_{+}$, a strict inequality holds between the values of $z$ in (5.12), except when $z\left(t^{\prime}\right)=z\left(t^{\prime \prime}\right)=0$. The exception is caused by the kink which $f(t, \cdot)$ has at $z=0$ (where the curve is "cut off" by setting $f(t, \mathbf{z})=-\infty$ for $\mathbf{z}<0)$ : see $(5.20)$.

Proof of Example 5.13. Take any $t \in T, \epsilon>0, p \in L^{1}(T)$ and $y$ that maximises $\langle p, \cdot\rangle$ on $S$, i.e., $z:=-y$ minimises $\langle p, \cdot\rangle$ on $-S=\{x: F(x) \geq \varphi\}$. There exists a scalar $\mu \geq 0$ such that

$$
\begin{aligned}
& p(\tau)=\mu \frac{\partial f}{\partial \mathbf{z}}(\tau, z(\tau)) \quad \text { for } \sigma \text {-almost every } \tau \in T \text { with } z(\tau)>0 \\
& p(\tau) \geq \mu \frac{\partial f}{\partial \mathbf{z}}(\tau, z(\tau)) \quad \text { for } \sigma \text {-almost every } \tau \in T \text { with } z(\tau)=0 .
\end{aligned}
$$

If $\mu>0$ then, by Corollary B.2 applied to $M=\mu \partial f / \partial z$, there exists an $H \in \mathcal{N}(t)$ such that: for every $t^{\prime}$ and $t^{\prime \prime}$ in $H$ and every $z^{\prime}$ and $z^{\prime \prime}$ in $[\operatorname{EssInf}(z), \operatorname{EssSup}(z)] \subset \mathbb{R}_{+}$

$$
\mathbf{z}^{\prime}<\mathbf{z}^{\prime \prime} \Rightarrow \frac{\partial f}{\partial \mathbf{z}}\left(t^{\prime}, \mathbf{z}^{\prime}\right) \geq \frac{\partial f}{\partial \mathbf{z}}\left(t^{\prime}, \mathbf{z}^{\prime \prime}\right) \geq \frac{\partial f}{\partial \mathbf{z}}\left(t^{\prime \prime}, \mathbf{z}^{\prime \prime}\right)-\frac{\epsilon}{\mu}
$$

\footnotetext{
${ }^{23}$ That the right (one-sided) derivative $(\partial f / \partial z)(t, 0)$ is finite is assumed only for simplicity. For the purpose of proving price continuity, a nondecreasing $f(t, \cdot)$ with $(\partial f / \partial z)(t, 0)=+\infty$ can be handled in the same way as $\partial u / \partial \mathrm{x}$ in Section 8; the infinite marginal productivity (at 0 ) only helps the argument (because it means that, like consumer demand, input demand responds to a price jump with a nonzero drop).
} 
From this and (5.19)-(5.20), for a.e. $t^{\prime}$ and $t^{\prime \prime}$ in $H$,

$$
z\left(t^{\prime}\right)<z\left(t^{\prime \prime}\right) \Rightarrow p\left(t^{\prime}\right) \geq \mu \frac{\partial f}{\partial \mathbf{z}}\left(t^{\prime}, z\left(t^{\prime}\right)\right) \geq \mu \frac{\partial f}{\partial \mathbf{z}}\left(t^{\prime \prime}, z\left(t^{\prime \prime}\right)\right)-\epsilon=p\left(t^{\prime \prime}\right)-\epsilon .
$$

In other words, for a.e. $t^{\prime}$ and $t^{\prime \prime}$ in $H$,

$$
\epsilon+p\left(t^{\prime}\right)<p\left(t^{\prime \prime}\right) \Rightarrow z\left(t^{\prime}\right) \geq z\left(t^{\prime \prime}\right)
$$

which proves (5.12), since $y=-z$.

Finally, if $\mu=0$ then $p\left(t^{\prime \prime}\right)>p\left(t^{\prime}\right)$ implies that $z\left(t^{\prime \prime}\right)=0 \leq z\left(t^{\prime}\right)$, as required.

There is an even weaker condition on the technologies which, together with subsymmetry of preferences, ensures price continuity in equilibrium. It holds for energy storage (Lemma 9.1). To formulate it, we use the concept of the essential value of $p$ at $t$, discussed in Appendix A. Denoted by ess $p(t)$, it exists if and only if the lower and upper (essential) values are equal and finite. In other words, $t \notin$ dom ess $p$ if and only if either $-\infty<\underline{p}(t)<\bar{p}(t)<+\infty$ or $\underline{p}(t)=-\infty$ or $\bar{p}(t)=+\infty$.

Definition 5.14. A set $S \subset L^{\infty}(T, \sigma)$ is quasi-symmetric if:

For every $p \in L^{1}(T)$ and every $y$ that maximises $\langle p, \cdot\rangle$ on $S$, and for any $t_{\ddagger} \in$ $T \backslash$ dom ess $p$, there is a number $\alpha>0$ such that every neighbourhood $N \in \mathcal{N}\left(t_{\ddagger}\right)$ has a pair of $\sigma$-nonnull subsets, $A^{\prime} \subset N$ and $A^{\prime \prime} \subset N$, such that

$$
\begin{array}{r}
\alpha+\operatorname{ess} \sup _{A^{\prime}} p \leq \operatorname{ess} \inf _{A^{\prime \prime}} p \\
\text { ess } \sup _{A^{\prime}} y \leq \operatorname{ess} \inf _{A^{\prime \prime}} y .
\end{array}
$$

i.e., for $\sigma$-almost every $t^{\prime} \in A^{\prime}$ and $t^{\prime \prime} \in A^{\prime \prime},{ }^{24}$

$$
\begin{aligned}
\alpha+p\left(t^{\prime}\right) & \leq p\left(t^{\prime \prime}\right) \\
y\left(t^{\prime}\right) & \leq y\left(t^{\prime \prime}\right) .
\end{aligned}
$$

Comments:

1. Again, with a quasi-symmetric $S$ the relationship between $p$ and $y$ is not symmetric: $p$ and $y$ cannot be interchanged in (5.21)-(5.22) or (5.23)-(5.24).

2. The strict inequality between the values of $p$ in (5.21) or (5.23) can be made nonstrict without changing the concept. But the inequality between the values of $y$ in (5.22) or (5.24) must be non-strict, as in (5.2) or (5.3).

The "price part" of the quasi-symmetry condition is always met because it follows purely from the hypothesis of a price discontinuity (i.e., from nonexistence of ess $p$ at $\left.t_{\ddagger}\right)$.

\footnotetext{
${ }^{24}$ This means "for every $t^{\prime}$ in $A^{\prime}$ and $t^{\prime \prime}$ in $A^{\prime \prime}$ but outside of some $\sigma$-null set $Z$ ". The excepted set $Z$ depends on the choice of variants of $p$ and $y$, but it can be chosen independently of $N$ because the topology of $T$ has a countable local base at each $t \in T$.
} 
Remark 5.15. For any $t_{\ddagger} \in T \backslash$ dom ess $p$, there is an $\alpha>0$ such that every $N \in$

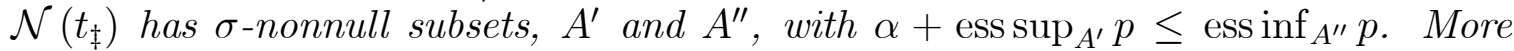
specifically:

1. If $\underline{p}\left(t_{\ddagger}\right)=-\infty$ or $\bar{p}\left(t_{\ddagger}\right)=+\infty$, then every $\alpha>0$ has this property.

2. If $-\infty<\underline{p}\left(t_{\ddagger}\right)<\bar{p}\left(t_{\ddagger}\right)<+\infty$, then any (positive) $\alpha<\bar{p}\left(t_{\ddagger}\right)-\underline{p}\left(t_{\ddagger}\right)$ will do.

Proof. For every $N \in \mathcal{N}\left(t_{\ddagger}\right)$

$$
\operatorname{ess} \inf _{N} p \leq \underline{p}\left(t_{\ddagger}\right) \quad \text { and } \quad \bar{p}\left(t_{\ddagger}\right) \leq \operatorname{ess} \sup _{N} p .
$$

Case 1: If $\bar{p}\left(t_{\ddagger}\right)=+\infty$ then $\operatorname{ess}_{\sup _{N}} p=+\infty$; so for every $\alpha$ there is an $A^{\prime \prime} \subset N$ with $p \geq \operatorname{essinf}_{N} p+1+\alpha$ almost everywhere (a.e.) on $A^{\prime \prime}$. There is also an $A^{\prime} \subset N$ with $p \leq \operatorname{ess~inf~}_{N} p+1$ a.e. on $A^{\prime}$; and hence (5.21).

When $p\left(t_{\ddagger}\right)=-\infty$, the argument is similar.

Case 2: If $-\infty<\underline{p}\left(t_{\ddagger}\right)<\bar{p}\left(t_{\ddagger}\right)<+\infty$, then $\operatorname{ess} \inf _{N_{0}} p$ and $\operatorname{ess}_{\sup _{N_{0}}} p$ are finite for some $N_{0} \in \mathcal{N}\left(t_{\ddagger}\right)$. For every $N \in \mathcal{N}\left(t_{\ddagger}\right)$ and $\epsilon>0$ there exist $A^{\prime}, A^{\prime \prime} \subset N \cap N_{0}$ such that

$$
p \leq \operatorname{ess} \inf _{N \cap N_{0}} p+\frac{\epsilon}{2} \quad \text { a.e. on } A^{\prime} \quad \text { and } \quad p \geq \operatorname{ess} \sup _{N \cap N_{0}} p-\frac{\epsilon}{2} \quad \text { a.e. on } A^{\prime \prime} .
$$

Used with an $\epsilon \leq \bar{p}\left(t_{\ddagger}\right)-\underline{p}\left(t_{\ddagger}\right)-\alpha$, this gives

$$
\alpha \leq \bar{p}\left(t_{\ddagger}\right)-\underline{p}\left(t_{\ddagger}\right)-\epsilon \leq \operatorname{ess} \sup _{N \cap N_{0}} p-\operatorname{ess} \inf _{N \cap N_{0}} p-\epsilon \leq \operatorname{ess} \inf _{A^{\prime \prime}} p-\operatorname{ess} \sup _{A^{\prime}} p
$$

as required.

Corollary 5.16. Every sub-symmetric set (and hence every symmetric set) is quasisymmetric.

Proof. Given a sub-symmetric $S \subset L^{\infty}(T)$ and any $p, t_{\ddagger}$ and $y$ as in Definition 5.14, fix any $\alpha>0$ that is less than $\bar{p}\left(t_{\ddagger}\right)-p\left(t_{\ddagger}\right)$ if $\bar{p}\left(t_{\ddagger}\right)$ and $\underline{p}\left(t_{\ddagger}\right)$ are finite. Fix any $\epsilon<\alpha$, and choose an $H \in \mathcal{N}\left(t_{\ddagger}\right)$ as in Definition 5.11. For every $N \in \mathcal{N}\left(t_{\ddagger}\right)$, apply Remark 5.15 (to $N \cap H$ ) to choose nonnull subsets, $A^{\prime}$ and $A^{\prime \prime}$, of $N \cap H$ with

$$
\epsilon+\operatorname{ess} \sup _{A^{\prime}} p<\alpha+\operatorname{ess} \sup _{A^{\prime}} p \leq \operatorname{ess} \inf _{A^{\prime \prime}} p .
$$

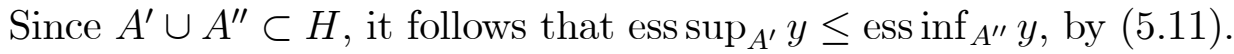

The condition formulated next is used in Section 8 to ensure, in the process of proving price continuity, that the price is bounded and that consumption is therefore bounded away from zero.

Definition 5.17. A set $S \subset L^{\infty}(T)$ is pseudo-symmetric if, for every $p \in L^{1}(T)$ and every $y$ that maximises $\langle p, \cdot\rangle$ on $S$,

$$
\lim _{\mathbf{p} \nearrow+\infty} \operatorname{essinf}_{t: p(t)>\mathbf{p}} y(t) \geq \operatorname{EssSup}(y):=\operatorname{ess} \sup _{t \in T} y(t)
$$


i.e., for every $\delta>0$ there is a $\mathrm{p} \in \mathbb{R}$ such that, for $\sigma$-almost every $t \in T,{ }^{25}$

$$
p(t)>\mathrm{p} \Rightarrow y(t) \geq \operatorname{EssSup}(y)-\delta .
$$

Comment: The condition is of interest only when $p$ is unbounded. For a $p \in L^{\infty}$, it is met vacuously by $\mathrm{p}=\operatorname{EssSup}(p)$ or larger (since this means that $p(t) \leq \mathrm{p}$ for a.e. $t)$.

Lemma 5.18. Every symmetric set is pseudo-symmetric.

Proof. Given a symmetric $S \subset L^{\infty}(T)$ and any $p$ and $y$ maximising $\langle p, \cdot\rangle$ on $S$, fix any $\delta>0$ and denote the set of $\delta$-near-peaks of $y$ by

$$
P_{\delta}(y):=\{t \in T: y(t) \geq \operatorname{EssSup}(y)-\delta\} .
$$

Take any two numbers $\mathrm{p}^{\prime \prime}$ and $\mathrm{p}^{\prime}$ with

$$
\mathrm{p}^{\prime \prime}>\mathrm{p}^{\prime}>\underset{P_{\delta}(y)}{\operatorname{essinf}} p
$$

and define the sets

$$
A^{\prime}:=\left\{t \in P_{\delta}: p(t) \leq \mathrm{p}^{\prime}\right\} \quad \text { and } \quad A^{\prime \prime}:=\left\{t \in T: p(t) \geq \mathrm{p}^{\prime \prime}\right\}
$$

Then

$$
\text { ess } \inf _{A^{\prime \prime}} p \geq \mathrm{p}^{\prime \prime}>\mathrm{p}^{\prime} \geq \operatorname{ess} \sup _{A^{\prime}} p
$$

which implies (by Lemma 5.9 and Definition 5.3) that

$$
\operatorname{ess} \inf _{A^{\prime \prime}} y \geq \operatorname{ess} \sup _{A^{\prime}} y \geq \operatorname{ess} \inf _{A^{\prime}} y \geq \operatorname{EssSup}(y)-\delta
$$

(with the penultimate inequality holding because $A^{\prime}$ is nonnull.) This means that (5.25) holds for $\mathrm{p}=\mathrm{p}^{\prime \prime}$ (or larger).

An industrial user of a differentiated good meets this condition if his production function is additively separable. ${ }^{26}$

Example 5.19. With the assumptions of Example 5.13 on the concave integral functional $F(z):=\int_{T} f(t, z(t)) \sigma(\mathrm{d} t)$ for $z \in L_{+}^{\infty}$, if additionally $\sup _{t \in T}(\partial f / \partial \mathrm{z})(t, 0)<$ $+\infty$ (as is the case when $T$ is compact and $\partial f / \partial \mathbf{z}$ is continuous in $t$ ), then the set $S=\{y \leq 0: F(-y) \geq \varphi\}$ is pseudo-symmetric for any $\varphi \in \mathbb{R}$ (provided that $F(z)>\varphi$ for some $z)$.

Proof. As in the Proof of Example 5.13, take a $z:=-y$ minimises $\langle p, \cdot\rangle$ on $-S=$ $\{z: F(z) \geq \varphi\}$ and a $\mu \in \mathbb{R}_{+}$satisfying (5.19)-(5.20). Set $\mathrm{p}:=\mu \sup _{t \in T}(\partial f / \partial z)(t, 0)$. Then $\mathrm{p} \geq \mu(\partial f / \partial \mathbf{z})(t, \mathrm{z})$ for every $t \in T$ and $\mathrm{z} \in \mathbb{R}_{+}$, and so

$$
p(t)>\mathrm{p} \Rightarrow z(t)=0 \leq \operatorname{EssInf}(z)
$$

\footnotetext{
${ }^{25}$ The excepted null subset of $T$ can be chosen independently of $\delta$.

${ }^{26}$ Similarly, a supplier of the good meets the condition if his cost is additively separable as in Example 5.12.
} 
which means that (5.25) holds even for $\delta=0$. (To prove this only for $\delta>0$, which is all that is required, it would suffice to assume $\sup _{t \in T}(\partial f / \partial z)(t, 0)<+\infty$ for every $z>0$.

The symmetry, sub-symmetry, quasi-symmetry and pseudo-symmetry concepts are applied to the sections of a production set $\mathbb{Y} \subset L^{\infty}(T) \times \mathbb{R}^{G}$ through a $q \in \mathbb{R}^{G}$. To say that such a set has symmetric (or sub-symmetric, etc.) $T$-sections means that for every $q \in \mathbb{R}^{G}$ the set $\mathbb{Y}(q)$ defined by (5.1) is symmetric (or sub-symmetric, etc.). These properties are mostly preserved in the summation of sets. To verify this, note first that

$$
\mathbb{Y}=\mathbb{Y}^{\prime}+\mathbb{Y}^{\prime \prime} \Rightarrow \mathbb{Y}(q)=\bigcup_{\left(q^{\prime}, q^{\prime \prime}\right): q^{\prime}+q^{\prime \prime}=q}\left(\mathbb{Y}^{\prime}\left(q^{\prime}\right)+\mathbb{Y}^{\prime \prime}\left(q^{\prime \prime}\right)\right) .
$$

Furthermore, the components of a profit-maximising $y \in \mathbb{Y}(q)$ are also profit maxima; i.e., if

$$
\begin{aligned}
& y=y^{\prime}+y^{\prime \prime} \text { maximises }\langle p, \cdot\rangle \text { on } \mathbb{Y}(q) \\
& q=q^{\prime}+q^{\prime \prime} \text { and }\left(y^{\prime}, q^{\prime}\right) \in \mathbb{Y}^{\prime} \text { and }\left(y^{\prime \prime}, q^{\prime \prime}\right) \in \mathbb{Y}^{\prime \prime}
\end{aligned}
$$

then

$$
y^{\prime} \text { maximises }\langle p, \cdot\rangle \text { on } \mathbb{Y}^{\prime}\left(q^{\prime}\right) \text { and } y^{\prime \prime} \text { maximises }\langle p, \cdot\rangle \text { on } \mathbb{Y}^{\prime \prime}\left(q^{\prime \prime}\right) \text {. }
$$

Lemma 5.20. If two subsets, $\mathbb{Y}^{\prime}$ and $\mathbb{Y}^{\prime \prime}$, of $L^{\infty}(T) \times \mathbb{R}^{G}$ have symmetric sections which are also convex and $\mathrm{w}\left(L^{\infty}, L^{1}\right)$-closed (weakly* closed) in $L^{\infty}(T)$, then $\mathbb{Y}:=$ $\mathbb{Y}^{\prime}+\mathbb{Y}^{\prime \prime}$ has symmetric sections.

Proof. This uses parts of the Hardy-Littlewood-Pólya Theorem, which characterises the majorisation order $\prec_{\text {HLP }}$, abbreviated to $\prec$ (defined below).

Take any $q \in \mathbb{R}^{G}, y \in \mathbb{Y}(q)$ and any $z \in L^{0}$ with the same distribution as $y$, i.e., $\sigma \circ z^{-1}=\sigma \circ y^{-1}$ (so $z \in L^{\infty}$ ). Since $y$ and $z$ have the same distribution (w.r.t. $\sigma$ ), there is a $\sigma$-doubly stochastic (d.s.) operator ${ }^{27} D$, on $L^{\infty}$, with $z=D y$ : see [24, Theorem 1]. ${ }^{28}$ Use (5.26) to choose $\left(y^{\prime}, q^{\prime}\right) \in \mathbb{Y}^{\prime}$ and $\left(y^{\prime \prime}, q^{\prime \prime}\right) \in \mathbb{Y}^{\prime \prime}$ with $y=y^{\prime}+y^{\prime \prime}$ and $q=q^{\prime}+q^{\prime \prime}$. Since $D$ is d.s., $D y^{\prime} \prec y^{\prime} \in \mathbb{Y}^{\prime}\left(q^{\prime}\right)$ : see, e.g., [4, 4.9] or [24, Theorem 3]. It follows that $D y^{\prime} \in \mathbb{Y}^{\prime}\left(q^{\prime}\right)$. (This step uses a characterisation of symmetry in terms of $\prec$, which

\footnotetext{
${ }^{27} \mathrm{~A} \sigma$-d.s. operator is also known as a Markov operator with $\sigma$ as an invariant measure.

${ }^{28}$ Denote by $D(\rho)^{*}$ the adjoint of $D(\rho) x:=x \circ \rho$, the d.s. operator associated with a measurepreserving map $\rho: T \rightarrow[0, \sigma(T)]$. (If $\rho$ is invertible, then $D(\rho)^{*}=D(\rho)^{-1}=D\left(\rho^{-1}\right)$, but $\rho$ may be noninvertible.) In these terms, a d.s. $D$ that maps $y$ to $z$ can be found as the composition $D=D\left(\rho^{\prime \prime}\right) D\left(\rho^{\prime}\right)^{*}$, for any $\rho^{\prime \prime} \in \mathcal{R}(z)$ and $\rho^{\prime} \in \mathcal{R}(y)$. This is because these inclusions mean that $z=D\left(\rho^{\prime \prime}\right) z_{\downarrow}$ and $y=D\left(\rho^{\prime}\right) y_{\downarrow}$, which implies that $y_{\downarrow}=D\left(\rho^{\prime}\right)^{*} y$ by $[24$, Lemma 3]; so, since $z_{\downarrow}=y_{\downarrow}$, it follows that $z=D\left(\rho^{\prime \prime}\right) D\left(\rho^{\prime}\right)^{*} y$. (The argument simplifies, of course, if $\rho^{\prime}$ is invertible: then $y_{\downarrow}=y \circ \rho^{\prime-1}$, so $z=y \circ \rho^{\prime-1} \circ \rho^{\prime \prime}$, and $D=D\left(\rho^{\prime \prime} \circ \rho^{\prime-1}\right)$ will do. $)$

Also, although both $D\left(\rho^{\prime \prime}\right)$ and $D\left(\rho^{\prime}\right)^{*}$ are extreme points of the convex set $\mathcal{D}$ of all d.s. operators [26, pp. 201-202], $D\left(\rho^{\prime \prime}\right) D\left(\rho^{\prime}\right)^{*}$ need not be extreme unless $\rho^{\prime \prime}$ or $\rho^{\prime}$ is invertible: see [26, Theorems 2 and 6]. However, the set $\mathcal{D}_{y, z}:=\{D \in \mathcal{D}: z=D y\}$ does contain an extreme point of $\mathcal{D}$ : see [26, Theorem 1].
} 
is the partial nonstrict preorder (a reflexive and transitive binary relation) defined on $L^{1}(T)$ by: $w \prec x$ if and only if $\int_{0}^{\tau} w_{\downarrow}(t) \mathrm{d} t \leq \int_{0}^{\tau} x_{\downarrow}(t) \mathrm{d} t$ for every $\tau \in[0, \sigma(T)]$, with equality when $\tau=\sigma(T) .{ }^{29}$ For $x_{\downarrow}$ see Definition 5.5. Denote the set of all functions on $T$ majorised by $x$ by $\operatorname{maj}(x):=\{w: w \prec x\}$, and denote the set of those equidistributed (a.k.a. equimeasurable) to $x$ by eqd $(x):=\left\{w: \sigma \circ w^{-1}=\sigma \circ x^{-1}\right\}$. If $x \in L^{\infty}(T)$ and $\sigma$ is nonatomic, then maj $(x)$ is convex and $\mathrm{w}\left(L^{\infty}, L^{1}\right)$-compact, and it is the $\mathrm{w}\left(L^{\infty}, L^{1}\right)$-closed convex hull of eqd $(x)$ : see, e.g., $[4,5.2] .{ }^{30}$ So a closed convex $S$ is symmetric if and only if the conditions $w \prec x \in S$ imply $w \in S$.)

As has been shown, $D y^{\prime} \in \mathbb{Y}^{\prime}\left(q^{\prime}\right)$. Similarly $D y^{\prime \prime} \in \mathbb{Y}^{\prime \prime}\left(q^{\prime \prime}\right)$. Hence $z=D y^{\prime}+D y^{\prime \prime} \in$ $\mathbb{Y}(q)$.

Comment: The relevant implication of symmetry is the similarity of arrangement for $p$ and $y \in \operatorname{argmax}_{\mathbb{Y}(q)}\langle p, \cdot\rangle$, by Lemma 5.9. Preservation of this property (in summation) is even simpler to prove than preservation of symmetry: if $y$ maximises $\langle p, \cdot\rangle$ on $\mathbb{Y}(q)$, use (5.26) to decompose it as in (5.27)-(5.28). Then, by (5.29) and the assumed property of $\mathbb{Y}^{\prime}\left(q^{\prime}\right)$ and $\mathbb{Y}^{\prime \prime}\left(q^{\prime \prime}\right)$, both $y^{\prime}$ and $y^{\prime \prime}$ are arranged similarly to $p$; and it follows that so is $y=y^{\prime}+y^{\prime \prime}$ (Remark 5.8). A similar argument, spelt out next, applies to the weaker conditions.

Lemma 5.21. For any two subsets, $\mathbb{Y}^{\prime}$ and $\mathbb{Y}^{\prime \prime}$, of $L^{0}(T) \times \mathbb{R}^{G}$ :

1. If both $\mathbb{Y}^{\prime}$ and $\mathbb{Y}^{\prime \prime}$ have sub-symmetric T-sections, then so has $\mathbb{Y}:=\mathbb{Y}^{\prime}+\mathbb{Y}^{\prime \prime}$.

2. If $\mathbb{Y}^{\prime}$ has quasi-symmetric sections, and $\mathbb{Y}^{\prime \prime}$ has sub-symmetric sections, then $\mathbb{Y}:=\mathbb{Y}^{\prime}+\mathbb{Y}^{\prime \prime}$ has quasi-symmetric sections.

3. If both $\mathbb{Y}^{\prime}$ and $\mathbb{Y}^{\prime \prime}$ have pseudo-symmetric sections, then so has $\mathbb{Y}:=\mathbb{Y}^{\prime}+\mathbb{Y}^{\prime \prime}$.

Proof. For all three parts of the lemma, given any $q \in \mathbb{R}^{G}$, any $p \in L^{1}(T)$ and $y$ that maximises $\langle p, \cdot\rangle$ on $\mathbb{Y}(q)$, use $(5.26)$ to choose $\left(y^{\prime}, q^{\prime}\right) \in \mathbb{Y}^{\prime}$ and $\left(y^{\prime \prime}, q^{\prime \prime}\right) \in \mathbb{Y}^{\prime \prime}$ with $y=y^{\prime}+y^{\prime \prime}$ and $q=q^{\prime}+q^{\prime \prime}$, as in (5.27)-(5.28). This ensures (5.29). From here on the proof depends on the part.

For Part 1, given also any $t \in T$ and $\epsilon>0$, use (5.29) and the sub-symmetry of both $\mathbb{Y}^{\prime}\left(q^{\prime}\right)$ and $\mathbb{Y}^{\prime \prime}\left(q^{\prime \prime}\right)$ to take $H^{\prime} \in \mathcal{N}(t)$ and $H^{\prime \prime} \in \mathcal{N}(t)$ as in Definition 5.11 with $y^{\prime}$ or $y^{\prime \prime}$ in place of $y$. Set $H:=H^{\prime} \cap H^{\prime \prime}$; then for $\sigma$-a.e. $t_{1}$ and $t_{2}$ in $H$, if $\epsilon+p\left(t_{1}\right)<p\left(t_{2}\right)$ then both $y^{\prime}\left(t_{1}\right) \leq y^{\prime}\left(t_{2}\right)$ and $y^{\prime \prime}\left(t_{1}\right) \leq y^{\prime \prime}\left(t_{2}\right)$, and so $y\left(t_{1}\right) \leq y\left(t_{2}\right)$ by adding up. This proves Part 1 .

For Part 2, one extends the Proof of Corollary 5.16 by combining it with the decomposition of $y$. Given also any $t_{\ddagger} \in T \backslash$ dom ess $p$, use (5.29) and the quasisymmetry of $\mathbb{Y}^{\prime}\left(q^{\prime}\right)$ to take an $\alpha>0$ as in Definition 5.14 (with $y^{\prime}$ in place of $y$ ). Fix any positive $\epsilon<\alpha$, and use (5.29) and the sub-symmetry of $\mathbb{Y}^{\prime \prime}\left(q^{\prime \prime}\right)$, to choose an $H \in \mathcal{N}\left(t_{\ddagger}\right)$ as in Definition 5.11 (with $y^{\prime \prime}$ in place of $y$ ). For every $N \in \mathcal{N}\left(t_{\ddagger}\right)$, by the

\footnotetext{
${ }^{29}$ Roughly speaking, $w \prec x$ means that the distribution of $w$ (w.r.t. $\sigma$ ) is "more concentrated about the average" than the distribution of $x$.

${ }^{30} \mathrm{~A}$ stronger result is that eqd $(x)$ is the set of all the extreme points of maj $(x)$ : see [25, p. 1026]. A similar result holds for weak majorisation [8].
} 
quasi-symmetry of $\mathbb{Y}^{\prime}\left(q^{\prime}\right)$, there exist nonnull subsets, $A_{1}$ and $A_{2}$, of $N \cap H$ with

$$
\begin{aligned}
& \operatorname{ess} \sup _{A_{1}} y^{\prime} \leq \operatorname{ess} \inf _{A_{2}} y^{\prime} \\
& \epsilon+\operatorname{ess} \sup _{A_{1}} p<\alpha+\operatorname{ess} \sup _{A_{1}} p \leq \operatorname{ess} \inf _{A_{2}} p .
\end{aligned}
$$

Since $A_{1} \cup A_{2} \subset H$, it follows - from (5.31) and (5.11) applied to $y^{\prime \prime} \in \mathbb{Y}^{\prime \prime}\left(q^{\prime \prime}\right)$ in place of $y \in S$ - that

$$
\operatorname{ess} \sup _{A_{1}} y^{\prime \prime} \leq \operatorname{ess} \inf _{A_{2}} y^{\prime \prime}
$$

Adding this to (5.30) and applying (5.9)-(5.10) completes the argument.

For Part 3, given also any $\delta>0$, use (5.29) and the pseudo-symmetry of both $\mathbb{Y}^{\prime}\left(q^{\prime}\right)$ and $\mathbb{Y}^{\prime \prime}\left(q^{\prime \prime}\right)$ to take $\mathrm{p}^{\prime \prime}$ and $\mathrm{p}^{\prime}$ such that, for a.e. $t$,

$$
\begin{aligned}
& p(t)>\mathbf{p}^{\prime} \Rightarrow y^{\prime}(t) \geq \operatorname{EssSup}\left(y^{\prime}\right)-\frac{\delta}{2} \\
& p(t)>\mathbf{p}^{\prime \prime} \Rightarrow y^{\prime \prime}(t) \geq \operatorname{EssSup}\left(y^{\prime \prime}\right)-\frac{\delta}{2}
\end{aligned}
$$

and set $\mathrm{p}=\max \left\{\mathrm{p}^{\prime}, \mathrm{p}^{\prime \prime}\right\}$. Then, for a.e. $t$,

$$
p(t)>\mathrm{p} \Rightarrow y(t)=y^{\prime}(t)+y^{\prime \prime}(t) \geq \operatorname{EssSup}\left(y^{\prime}\right)+\operatorname{EssSup}\left(y^{\prime \prime}\right)-\delta \geq \operatorname{EssSup}(y)-\delta
$$

as required.

\section{Sub-Symmetry of PREFEREnCES}

A variant of the sub-symmetry concept is needed to formulate a condition on consumer preferences which, together with quasi-symmetry of the production set, ensures price continuity in equilibrium. For use in this context, the condition is reoriented to minimisation of "expenditure" instead of maximisation of "profit" as in Definition 5.11. (Also, it is formulated "pointwise" because its verification requires a condition on the $x$ in question, e.g., that EssInf $(x)>0$ in Example 6.2. This is needed because the demand $x(t)$ cannot drop when it is zero.) Recall that $\mathcal{N}(t)$ means the set of all neighbourhoods of $t$.

Definition 6.1. A set $S \subset L^{\infty}(T)$ is strongly sub-symmetric at a point $x \in S$ if: For every $p \in L^{1}(T)$ such that $x$ minimises $\langle p, \cdot\rangle$ on $S$, and for every $t \in T$ and $\epsilon>0$, there exist an $H \in \mathcal{N}(t)$ and $a \delta>0$ such that for any two measurable sets $A^{\prime} \subset H$ and $A^{\prime \prime} \subset H$

$$
\epsilon+\operatorname{ess} \sup _{A^{\prime}} p<\operatorname{ess} \inf _{A^{\prime \prime}} p \Rightarrow \operatorname{ess} \inf _{A^{\prime}} x \geq \delta+\operatorname{ess} \sup _{A^{\prime \prime}} x .
$$

Equivalently, for any $t$ and $\epsilon$ such as above, there exists an $H \in \mathcal{N}(t)$ and $a \delta>0$ such that, for $\sigma$-almost every $t^{\prime}$ and $t^{\prime \prime}$ in $H$,

$$
\epsilon+p\left(t^{\prime}\right)<p\left(t^{\prime \prime}\right) \Rightarrow x\left(t^{\prime}\right) \geq x\left(t^{\prime \prime}\right)+\delta \text {. }
$$




\section{Comments:}

1. If $S$ is strongly sub-symmetric (at every $x$ ), then it is sub-symmetric.

2. A symmetric set need not be strongly sub-symmetric at every point. For example, if $c(t, y(t))$ in (5.13) is $c(y(t))$, a convex function of $y(t)$ alone, then the sublevel set (5.14) is symmetric and hence sub-symmetric, but not strongly so, unless $c$ is differentiable.

3. In (6.1) or (6.2), the inequalities can be made nonstrict or strict without changing the concept (because of the $\epsilon$ and $\delta$ ).

This concept is used with $S$ equal to a superlevel set for the ordering of $L_{+}^{\infty}(T)$ obtained by fixing an $\widetilde{m} \in \mathbb{R}^{G}$ in an ordering $\preccurlyeq$ of $L_{+}^{\infty}(T) \times \mathbb{R}_{+}^{G}$, i.e., with $S$ equal to

$$
S(\widetilde{x}, \widetilde{m}, \preccurlyeq):=\left\{x \in L_{+}^{\infty}(T):(\widetilde{x}, \widetilde{m}) \preccurlyeq(x, \widetilde{m})\right\},
$$

a "preferred set" for an $\widetilde{m}$-section of $\preccurlyeq$. When $\preccurlyeq$ is defined by a function $\mathbb{U}$ on $L_{+}^{\infty}(T) \times \mathbb{R}_{+}^{G}$, this set is $\{x: \mathbb{U}(\widetilde{x}, \widetilde{m}) \leq \mathbb{U}(x, \widetilde{m})\}$, a superlevel set of $U=\mathbb{U}(\cdot, \widetilde{m})$.

An example of strong sub-symmetry in consumption is the additively separable utility function $U(x)=\int_{T} u(t, x(t)) \mathrm{d} t$ (for a fixed $m$ ). This is mathematically similar to the case of additively separable cost and production functions (Examples 5.12 and $5.13)$.

Example 6.2. Assume that $u: T \times \mathbb{R}_{+} \rightarrow \mathbb{R}$ is a differentiable concave integrand, i.e., the function $t \mapsto u(t, \mathrm{x})$ is $\sigma$-integrable on $T$ (for every $\mathrm{x} \in \mathbb{R}_{+}$), whilst the function $\mathrm{x} \mapsto u(t, \mathrm{x})$ is concave, (strictly) increasing and differentiable on $\mathbb{R}_{++}$(and continuous also at $\mathrm{x}=0$ ), for every $t \in T$. Then

$$
U(x):=\int_{T} u(t, x(t)) \sigma(\mathrm{d} t)
$$

is a concave integral functional on $L_{+}^{\infty}(T)$ : see, e.g., [22].

If additionally $\partial u / \partial \times$ is (jointly) continuous on $T \times \mathbb{R}_{++}$, then, for any $\widetilde{x} \in L^{\infty}(T)$ with $\operatorname{EssInf}(\widetilde{x})>0$, the set

$$
S=\left\{x \in L_{+}^{\infty}(T): U(x) \geq U(\widetilde{x})\right\}
$$

is strongly sub-symmetric at $\widetilde{x}$.

Proof. Take any $t \in T, \epsilon>0$ and $p \in L^{1}(T)$ such that $x$ minimises $\langle p, \cdot\rangle$ on $S$ (so $p \geq 0$ ). If $p=0$, there is nothing to prove. If $p \neq 0$, then (since $\operatorname{EssInf}(\widetilde{x})>0$ ) there 
is a (unique) scalar $\widetilde{\lambda}>0$ such that, for a.e. $t \in T,{ }^{31}$

$$
\widetilde{\lambda} p(t)=\frac{\partial u}{\partial \mathbf{x}}(t, \widetilde{x}(t)) .
$$

Since $\operatorname{EssInf}(\widetilde{x})>0$, Corollary B.2 applies to $M=\partial u_{h} / \partial \mathrm{x}$ with $\underline{\mathrm{x}}:=\operatorname{EssInf}(\widetilde{x})$ and $\bar{x}:=\operatorname{EssSup}(\widetilde{x})$; so for every $t \in T$ and $\epsilon>0$ there exist a number $\delta>0$ and an $H \in \mathcal{N}(t)$ such that: for each $h$, every $t^{\prime}$ and $t^{\prime \prime}$ in $H$ and every $\mathrm{x}^{\prime}$ and $\mathrm{x}^{\prime \prime}$ in $[\underline{\mathrm{x}}, \overline{\mathrm{x}}] \subset \mathbb{R}_{++}$

$$
\mathrm{x}^{\prime}<\mathrm{x}^{\prime \prime}+\delta \Rightarrow \frac{\partial u}{\partial \mathbf{x}}\left(t^{\prime}, \mathrm{x}^{\prime}\right)>\frac{\partial u}{\partial \mathrm{x}}\left(t^{\prime \prime}, \mathrm{x}^{\prime \prime}\right)-\tilde{\lambda} \epsilon
$$

From this and (6.6), for a.e. $t^{\prime}$ and $t^{\prime \prime}$ in $H$,

$$
\widetilde{x}\left(t^{\prime}\right)<\widetilde{x}\left(t^{\prime \prime}\right)+\delta \Rightarrow p\left(t^{\prime}\right)=\frac{1}{\widetilde{\lambda}} \frac{\partial u_{h}}{\partial \mathrm{x}}\left(t^{\prime}, \widetilde{x}\left(t^{\prime}\right)\right)>\frac{1}{\widetilde{\lambda}} \frac{\partial u}{\partial \mathrm{x}}\left(t^{\prime \prime}, \widetilde{x}\left(t^{\prime \prime}\right)\right)-\epsilon=p\left(t^{\prime \prime}\right)-\epsilon .
$$

In other words, for a.e. $t^{\prime}$ and $t^{\prime \prime}$ in $H$,

$$
\epsilon+p\left(t^{\prime}\right) \leq p\left(t^{\prime \prime}\right) \Rightarrow \widetilde{x}\left(t^{\prime}\right) \geq \widetilde{x}\left(t^{\prime \prime}\right)+\delta
$$

which proves $(6.2)$.

The strong sub-symmetry condition can be verified for other functional forms of utility. For example, the additively separable form can be generalised by adding further terms.

Example 6.3. Assume that $v: T \times \mathbb{R}_{+} \times T \times \mathbb{R}_{+} \rightarrow \mathbb{R}$ has the properties:

1. The function $(\mathrm{x}, \xi) \mapsto u(t, \mathrm{x}, \tau, \xi)$ is jointly concave, increasing and continuously differentiable on $\mathbb{R}_{+}^{2}$ (for every $(t, \tau) \in T \times T$ ).

2. The function $(t, \tau) \mapsto v(t, \mathrm{x}, \tau, \xi)$ is $\sigma \times \sigma$-integrable on $T \times T$ (for every $(\mathrm{x}, \xi) \in$ $\left.\mathbb{R}_{+}^{2}\right)$.

With $u: T \times \mathbb{R}_{+} \rightarrow \mathbb{R}$ as in Example 6.2 (and $\partial u / \partial \times$ continuous), define

$$
U(x):=\int_{T} u(t, x(t)) \sigma(\mathrm{d} t)+\int_{T} \int_{T} v(t, x(t), \tau, x(\tau)) \sigma(\mathrm{d} t) \sigma(\mathrm{d} \tau)
$$

for $x \in L_{+}^{\infty}(T)$. Assume also, without loss of generality, that $v(t, \mathrm{x}, \tau, \xi)=v(\tau, \xi, t, \mathrm{x})$. If additionally $\partial^{2} v / \partial \times \partial \xi \leq 0$ everywhere and $\operatorname{EssInf}(\widetilde{x})>0$, then the set

$$
S=\left\{x \in L_{+}^{\infty}(T): U(x) \geq U(\widetilde{x})\right\}
$$

\footnotetext{
${ }^{31}$ To prove (6.6), use the formula for the normal cone to a superlevel set of a continuous concave function: see, e.g., [17, 4.3: Proposition 2] or [28, 7.8]. (Slater's Condition holds here because $u$ is increasing in $\mathrm{x}$; and $U$ is norm-continuous at $\widetilde{x}$ because $\operatorname{EssInf}(\widetilde{x})>0$.) This shows that $p \in \lambda^{-1} \partial U(\widetilde{x})$ for some $\lambda>0$; and the formula for $\partial U$-given in [22, Corollary 2C] and [17, 8.3: Theorem 3]-completes the argument. (Here $\partial U$ reduces to $\nabla U$.) It also follows that $p \gg 0$.

Given Remark 8.2, the FOC for an expenditure minimum can also be deduced from the FOC for a utility maximum, i.e., from Lemma 8.1 with $G=0$. (This applies here because $\langle p, \widetilde{x}\rangle>0$.)
} 
is strongly sub-symmetric at $\widetilde{x} .^{32}$

\section{Continuity of the equilibrium price Density function}

As is shown next, the weak "symmetry-like" conditions are sufficient for price continuity in equilibrium. The sets of producers and households (or consumers) are denoted by $\operatorname{Pr}$ and Ho. The production set of producer $i \in \operatorname{Pr}$ is $\mathbb{Y}_{i} \subset L^{\infty}(T) \times \mathbb{R}^{G}$, and $\mathbb{Y}:=\sum_{i \in \operatorname{Pr}} \mathbb{Y}_{i}$ is the total production set. The consumption set of each household $h \in$ Ho is the nonnegative orthant $L_{+}^{\infty}(T) \times \mathbb{R}_{+}^{G}$. Consumer preferences, taken to be complete and transitive, are given by a total (a.k.a. complete) weak preorder $\preccurlyeq_{h}$. The corresponding strict preference is denoted by $\prec_{h}$. The household's initial endowment is denoted by $\left(x_{h}^{\mathrm{En}}, m_{h}^{\mathrm{En}}\right) \in L_{+}^{\infty} \times \mathbb{R}_{+}^{G}$; and the household's share in the profits of producer $i$ is $\varsigma_{h i} \geq 0$, with $\sum_{h} \varsigma_{h i}=1$ for each $i$. (The ranges of running indices in summations, etc., are always taken to be the largest possible with any specified restrictions.)

Definition 7.1. A price system $\left(p^{\star}, r^{\star}\right) \in L^{1} \times \mathbb{R}^{G}$ supports an allocation, $\left(x_{h}^{\star}, m_{h}^{\star}\right) \geq$ 0 and $\left(y_{i}^{\star}, q_{i}^{\star}\right) \in \mathbb{Y}_{i}$ for each $h \in$ Ho and $i \in \operatorname{Pr}$, as a competitive equilibrium if:

1. $\sum_{h}\left(x_{h}^{\star}-x_{h}^{\mathrm{En}}, m_{h}^{\star}-m_{h}^{\mathrm{En}}\right)=\left(y^{\star}, q^{\star}\right):=\sum_{i}\left(y_{i}^{\star}, q_{i}^{\star}\right)$.

2. $\left\langle p^{\star}, y_{i}^{\star}\right\rangle+\left\langle r^{\star}, q_{i}^{\star}\right\rangle=\sup _{y, q}\left\{\left\langle p^{\star}, y\right\rangle+\left\langle r^{\star}, q\right\rangle:(y, q) \in \mathbb{Y}_{i}\right\}$.

3. $\left\langle p^{\star}, x_{h}^{\star}\right\rangle+\left\langle r^{\star}, m_{h}^{\star}\right\rangle=\left\langle p^{\star}, x_{h}^{\text {En }}+\sum_{i} \varsigma_{h i} y_{i}^{\star}\right\rangle+\left\langle r^{\star}, m_{h}^{\text {En }}+\sum_{i} \varsigma_{h i} q_{i}^{\star}\right\rangle$.

4. For every $(x, m) \geq 0$, if $\left\langle p^{\star}, x\right\rangle+\left\langle r^{\star}, m\right\rangle \leq\left\langle p^{\star}, x_{h}^{\star}\right\rangle+\left\langle r^{\star}, m_{h}^{\star}\right\rangle$, then $(x, m) \preccurlyeq h$ $\left(x_{h}^{\star}, m_{h}^{\star}\right)$.

Theorem 7.2. Assume that:

1. A price system $\left(p^{\star}, r^{\star}\right) \in L^{1}(T, \sigma) \times \mathbb{R}^{G}$ supports a competitive equilibrium with a consumption allocation $\left(x_{h}^{\star}, m_{h}^{\star}\right) \in L_{+}^{\infty}(T) \times \mathbb{R}_{+}^{G}$ (for $h \in \mathrm{Ho}$ ) and with a total input-output bundle $\left(y^{\star}, q^{\star}\right) \in \mathbb{Y}$.

2. The section $\mathbb{Y}\left(q^{\star}\right)$ of the total production set is a quasi-symmetric subset of $L^{\infty}(T)$.

3. The set $S\left(x_{h}^{\star}, m_{h}^{\star}, \preccurlyeq h\right)$, defined by (6.3), is strongly sub-symmetric at $x_{h}^{\star}$, for each household $h$.

4. $x_{h}^{\mathrm{En}} \in \mathcal{C}_{+}(T)$ for each $h$ (i.e., the initial endowment is nonnegative and has a continuous variant).

Then the equilibrium price has a continuous variant, viz., ess $p^{\star} \in \mathcal{C}(T)$.

Proof. Recall that $T$ has a countable base of open sets which are $\sigma$-nonnull. So, by Corollary A.3, it suffices to show that ess $p^{\star}$ exists everywhere on $T$. Suppose contrarily that there is a $t \in T \backslash$ dom ess $p^{\star}$. Since $y^{\star}$ maximises $\left\langle p^{\star}, \cdot\right\rangle$ on $Y\left(q^{\star}\right)$, use the quasi-symmetry of $Y\left(q^{\star}\right)$ to choose a number $\alpha>0$ as in Definition 5.14. Fix also

\footnotetext{
${ }^{32} \mathrm{~A}$ weaker sufficient condition on the derivatives is that $\partial^{2} v / \partial \mathrm{x} \partial \xi<\partial^{2} u / \partial \mathrm{x}^{2}$.
} 
any positive $\epsilon \leq \alpha .{ }^{33}$ Since $x_{h}^{\star}$ minimises $\left\langle p^{\star}, \cdot\right\rangle$ on $S\left(x_{h}^{\star}, m_{h}^{\star}, \preccurlyeq h\right),{ }^{34}$ use the strong sub-symmetry of this set to choose a number $\delta>0$ and an $H \in \mathcal{N}(t)$ such that, for each $h$ and for $\sigma$-almost every $t^{\prime}$ and $t^{\prime \prime}$ in $H$

$$
\epsilon+p^{\star}\left(t^{\prime}\right)<p^{\star}\left(t^{\prime \prime}\right) \Rightarrow x_{h}^{\star}\left(t^{\prime}\right) \geq x_{h}^{\star}\left(t^{\prime \prime}\right)+\delta .
$$

Fix any positive number $\beta \leq \delta$. By the continuity of $x_{h}^{\mathrm{En}}$, there is an $I \in \mathcal{N}(t)$ such that for a.e. $t^{\prime}$ and $t^{\prime \prime}$ in $I$, and for each $h$,

$$
\left|x_{h}^{\text {En }}\left(t^{\prime}\right)-x_{h}^{\text {En }}\left(t^{\prime \prime}\right)\right|<\beta \leq \delta .
$$

By the quasi-symmetry of $Y\left(q^{\star}\right)$ - i.e., by (5.21)-(5.22) applied to $N=H \cap I$ - there exist $\sigma$-nonnull sets $A^{\prime} \subset H \cap I$ and $A^{\prime \prime} \subset H \cap I$ with

$$
\begin{aligned}
\operatorname{ess} \sup _{A^{\prime}} y^{\star} & \leq \operatorname{ess} \inf _{A^{\prime \prime}} y^{\star} \\
\alpha+\operatorname{ess} \sup _{A^{\prime}} p^{\star} & \leq \operatorname{ess} \inf _{A^{\prime \prime}} p^{\star} .
\end{aligned}
$$

From this and (7.1) it follows that, for each $h$,

$$
x_{h}^{\star}\left(t^{\prime}\right) \geq x_{h}^{\star}\left(t^{\prime \prime}\right)+\delta \quad \text { for a.e. } t^{\prime} \in A^{\prime} \text { and } t^{\prime \prime} \in A^{\prime \prime}
$$

(i.e., for every $t^{\prime} \in A^{\prime} \backslash Z$ and every $t^{\prime \prime} \in A^{\prime \prime} \backslash Z$, where $Z$ is some $\sigma$-null set). Since $y^{\star}=\sum_{h}\left(x_{h}^{\star}-x_{h}^{\text {En }}\right)$, it follows from $(7.2)$ and (7.5) that $y^{\star}\left(t^{\prime}\right)>y^{\star}\left(t^{\prime \prime}\right)$ for a.e. $t^{\prime} \in A^{\prime}$ and $t^{\prime \prime} \in A^{\prime \prime}$; and a fortiori (since $\sigma\left(A^{\prime}\right)>0$ and $\sigma\left(A^{\prime \prime}\right)>0$ )

$$
\text { ess } \sup _{A^{\prime}} y^{\star}>\operatorname{ess} \inf _{A^{\prime \prime}} y^{\star} \text {. }
$$

This contradicts (7.3) and thus proves that dom ess $p^{\star}=T$.

\section{Continuity And Boundedness of PRICE AND QuANTITY With AdDitively SEPARABLE UTILITY}

The strong sub-symmetry condition on preferences in Theorem 7.2 is met when consumer utility is a differentiable additively separable function on $L_{+}^{\infty}(T)$ - or, more precisely, when the utility from $x$ is a monotone function of an integral functional of $x$ (for a fixed $m$ ). Verification of sub-symmetry rests on Example 6.2 and the first-order condition, given next, for utility maximisation or expenditure minimisation.

Lemma 8.1. Assume that $u: T \times \mathbb{R}_{+} \times \mathbb{R}_{+}^{G} \rightarrow \mathbb{R}$ is a differentiable concave integrand parameterised by $\mathbb{R}_{+}^{G}$, i.e.:

1. For every $t \in T$, the function $(\mathrm{x}, m) \mapsto u(t, \mathrm{x}, m)$ is concave; and for every $m \in \mathbb{R}_{+}^{G}$ :

2. The function $t \mapsto u(t, \mathrm{x}, m)$ is $\sigma$-integrable on $T$ (for every $\mathrm{x} \in \mathbb{R}_{+}$).

\footnotetext{
${ }^{33}$ The value $\epsilon=\alpha$ will do, but a smaller $\epsilon$ dispels any impression that the argument "hangs by a thread", in the sense of relying on the contradiction between a strict inequality $a<b$ and the reverse inequality $a \geq b$. The same goes for the choice of a $\beta \leq \delta$.

${ }^{34}$ With $\preccurlyeq_{h}$ locally nonsatiated, utility maximisation implies expenditure minimisation: see Remark 8.2.
} 
3. The function $\mathrm{x} \mapsto u(t, \mathrm{x}, m)$ is concave, (strictly) increasing and continuous on $\mathbb{R}_{+}$, for every $t \in T$.

Assume also that $W: \mathbb{R}^{1+G} \rightarrow \mathbb{R}$ is differentiable, concave, and increasing (in each variable); and define

$$
\mathbb{U}(x, m):=W(U(x, m), m)
$$

where, for $x \in L_{+}^{\infty}(T)$ and $m \in \mathbb{R}_{+}^{G}$,

$$
U(x, m):=\int_{T} u(t, x(t), m) \sigma(\mathrm{d} t)
$$

(with $u(t, \mathrm{x}, m):=-\infty$ for $\mathrm{x}<0$ and $\mathbb{U}(x, m):=-\infty$ for $(x, m) \ngtr 0)$. If additionally $p \in L^{1}(T), M>0$, and $(\widetilde{x}, \widetilde{m})$ maximises $\mathbb{U}(x, m)$ over $x$ and $m$ subject to: $\langle p, x\rangle+$ $r \cdot m \leq M, x \geq 0$ and $m \geq 0$, then there exists a scalar $\tilde{\lambda}>0$ such that

$$
\widetilde{\lambda} p(t) \in \partial_{\mathrm{x}} u(t, \widetilde{x}(t), \widetilde{m}) \quad \text { for } \sigma \text {-almost every } t \in T \text {. }
$$

When $(\partial u / \partial \mathbf{x})(0)=+\infty$, it follows that $\widetilde{x} \gg 0$ (i.e., $\widetilde{x}(t)>0$ for a.e. $t \in T$ ). If additionally $u$ is differentiable w.r.t. $\mathrm{x}$ on $\mathbb{R}_{++}$, then there is a unique scalar $\widetilde{\lambda}>0$ such that, for a.e. $t \in T$,

$$
\widetilde{\lambda} p(t)=\frac{\partial u}{\partial \mathbf{x}}(t, \widetilde{x}(t), \widetilde{m}) .
$$

Proof. One way to derive (8.4) is to use the first-order condition of smooth optimisation, after extending $u(t, \cdot, m)$ to a differentiable function on the whole of $\mathbb{R}$. A more general method, which applies also when $u$ is nondifferentiable in $\mathrm{x}$, is the Kuhn-Tucker Condition of convex optimisation. (This does not require a concave extension of $u(t, \cdot, m)$ beyond $\mathbb{R}_{+}$, so it applies also when $\partial u / \partial \mathbf{x} \nearrow+\infty$ as $\mathbf{x} \searrow 0$, as is assumed from $(8.13)$ on.) For an $(\widetilde{x}, \widetilde{m}) \geq 0$ to be a maximum point it is necessary (and sufficient) that there exists a $(\lambda, \mu, \nu) \in \mathbb{R}_{++} \times L_{+}^{\infty *} \times \mathbb{R}_{+}^{G}$ such that, with $\partial$ denoting the subdifferential, ${ }^{35}$

$$
\begin{aligned}
(\lambda p-\mu, \lambda r-\nu) & \in \partial_{x, m} \mathbb{U}(\widetilde{x}, \widetilde{m}) \\
\langle p, \widetilde{x}\rangle+r \cdot \widetilde{m} & =M
\end{aligned}
$$

$\mu$ is concentrated on $\{t \in T: \widetilde{x}(t) \leq \epsilon\}$ for every $\epsilon>0$.

$$
\nu \cdot \widetilde{m}=0 .
$$

(See, e.g., [23, Examples 4', 4"], ${ }^{36}$ where [23, (8.12)] implies, through [23, Theorems 18 (a) and 17 (a)], "the strong form of the Kuhn-Tucker Theorem", i.e., the equivalence in [23, Corollary 15A], so that every primal optimum is "supported" by a dual optimum. And, with $M>0$, the Generalised Slater's Condition of [23, (8.12)] is verified at, e.g., the point $(x, m)=\left(\epsilon 1_{T} ; \epsilon, \ldots, \epsilon\right)$ : for sufficiently small $\epsilon>0$, it meets the budget

\footnotetext{
${ }^{35}$ Since $U$ is concave and $W$ is concave and increasing, their composition $\mathbb{U}$ is also concave.

${ }^{36}$ The "cone model" of inequality constraints [23, Examples 4', 4"] is also expounded in, e.g., [2, $4.2]$.
} 
constraint strictly, and it lies in the interior of $L_{+}^{\infty}(T) \times \mathbb{R}_{+}^{G}$ for the supremum norm. Finally, it is impossible for $\lambda$ to be 0 because of nonsatiation, i.e., because $u$ is increasing in $\mathrm{x}$, and $W$ is increasing in $U$.) Obviously (8.5) implies that

$$
\lambda p-\mu \in \partial_{x} \mathbb{U}(\widetilde{x}, \widetilde{m})=\frac{\partial W}{\partial U} \partial_{x} U(\widetilde{x}, \widetilde{m}) .
$$

From this and the formula for $\partial_{x} U$ given in, e.g., [22, Corollary $\left.2 \mathrm{C}\right]$ and $[17,8.3$ : Theorem 3] it follows that (with $\widetilde{m}$ suppressed from the notation)

$$
(\lambda p-\mu)_{\mathrm{CA}}(t)=\lambda p-\mu_{\mathrm{CA}}(t) \in \gamma \partial_{\mathrm{x}} u(t, \widetilde{x}(t)) \quad \text { for a.e. } t \in T
$$

where $\gamma:=\partial W / \partial U>0$, and $\mu_{\mathrm{CA}}$ is the countably additive part of $\mu \in L^{\infty *}$ (in the Hewitt-Yosida decomposition, for which see, e.g., [1, Appendix I: (26)-(27)] or [6, III.7.8]). Since the term $\mu_{\mathrm{CA}}(t)$ is nonnegative and actually vanishes unless $\widetilde{x}(t)=0$, it can be absorbed into $\partial_{\mathrm{x}} u(t, 0)=[(\partial u / \partial \mathbf{x})(t, 0),+\infty)$; and thus (8.10) can be restated as (8.3) with $\widetilde{\lambda}:=\lambda / \gamma$. When $\partial u / \partial \mathrm{x}=+\infty$ at $\mathrm{x}=0$, it follows that $\widetilde{x} \gg 0$ because $\partial_{\mathrm{x}} u=\emptyset$ at $\mathrm{x}=0$. When $u$ is additionally differentiable w.r.t. $\mathrm{x}$ on $\mathbb{R}_{++},(8.3)$ simplifies to $(8.4)$, since $\partial_{\mathrm{x}} u(t, \widetilde{x}(t))=\{(\partial u / \partial \mathbf{x})(t, \widetilde{x}(t))\}$.

\section{Comments:}

1. Formulae (8.3) and (8.4) can also be derived directly by subdifferentiation of $\mathbb{U}(x, m)-\delta((x, m) \mid \mathrm{H})$, where $\delta(\cdot \mid \mathrm{H})$ is the $0-\infty$ indicator function of the half-space

$$
\mathrm{H}(p, r ; M)=\left\{(x, m) \in L^{\infty} \times \mathbb{R}^{G}:\langle p, x\rangle+r \cdot m \leq M\right\} .
$$

(The constraint $(x, m) \geq 0$ is included in the definition of $\mathbb{U}$.) A point $(\widetilde{x}, \widetilde{m})$ yields the maximum in question if and only if

$$
\left.0 \in \partial_{x, m}(\mathbb{U}(x, m)+\delta((x, m) \mid \mathrm{H}(p, r ; M)))\right|_{\substack{x=\widetilde{x} \\ m=\widetilde{m}}} ^{\substack{x \\ \text { (n) }}}
$$

and this condition can be expanded, as in [23, p. 57] or [28, 7.2 and 7.9], by using the additivity of $\partial$ (for which see, e.g., [23, Theorem 20] or [28, 5.38 and $7.2]$ ). As before, the calculation is completed by the formula for $\partial_{x} U$ (in [22, Corollary 2C] and [17, 8.3: Theorem 3]).

2. That $p \in L^{1}$ is part of (8.4), but that part is assumed and not proved in Lemma 8.1: unless $\operatorname{EssInf}(\widetilde{x})>0, \widetilde{x}$ can be optimal also when $p \in L^{\infty *} \backslash L^{1}$.

Utility maximisation is usually equivalent to expenditure minimisation.

Remark 8.2. Assume that $\preccurlyeq$ is a total preorder on a closed convex subset $X$ of a real topological vector space $(L, \mathcal{T})$, and that $\preccurlyeq$ is $\mathcal{T}$-locally nonsatiated and lower semicontinuous along each linear segment of $X .{ }^{37}$ Then the following three conditions

\footnotetext{
${ }^{37}$ By definition, $\preccurlyeq$ is $\mathcal{T}$-locally nonsatiated if $x^{\prime} \in \operatorname{cl}_{\mathcal{T}}\left\{x \in X: x^{\prime} \prec x\right\}$ for every $x^{\prime} \in X$ (where $x^{\prime} \prec x$ means that $x^{\prime} \preccurlyeq x$ and $\left.x^{\prime} \nsucc x\right)$. Even for the strongest choice of $\mathcal{T}$ this follows from nonsatiation if $\preccurlyeq$ can be represented by a concave function $U$. Also, the line-wise l.s. continuity follows from l.s. continuity for any vector topology on $L$.
} 
on a point $\widetilde{x} \in X$ and a continuous linear functional $p \in(L, \mathcal{T})^{*}$ are equivalent to one another, provided that there exists an $x^{0} \in X$ with $\left\langle p, x^{0}\right\rangle<\langle p, \widetilde{x}\rangle$ :

1. For every $x \in X$, if $\langle p, x\rangle \leq\langle p, \widetilde{x}\rangle$ then $x \preccurlyeq \widetilde{x}$.

2. For every $x \in X$, if $\langle p, x\rangle<\langle p, \widetilde{x}\rangle$ then $x \preccurlyeq \widetilde{x}$.

3. For every $x \in X$, if $\langle p, x\rangle<\langle p, \widetilde{x}\rangle$ then $x \prec \widetilde{x}$ (i.e., if $x \succcurlyeq \widetilde{x}$, then $\langle p, x\rangle \geq$ $\langle p, \widetilde{x}\rangle)$.

Proof. Either of Conditions 1 and 3 obviously implies Condition 2. To show that Condition 2 implies Condition 3, take any $x^{\prime} \succcurlyeq \widetilde{x}$. Then by local nonsatiation (and by Condition 2)

$$
\left\langle p, x^{\prime}\right\rangle \geq \inf _{x}\left\{\langle p, x\rangle: x \succ x^{\prime}\right\} \geq\langle p, \widetilde{x}\rangle .
$$

Finally, to show that Condition 2 implies Condition 1 , take any $x \in X$ with $\langle p, x\rangle \leq$ $\langle p, \widetilde{x}\rangle$, and introduce $x^{\alpha}:=(1-\alpha) x^{0}+\alpha x$ for $\alpha \in[0,1)$. Then $\left\langle p, x^{\alpha}\right\rangle\langle\langle p, \widetilde{x}\rangle$, so $x^{\alpha} \preccurlyeq \widetilde{x}$ (by Condition 2). As $\alpha \nearrow 1$, it follows that $x \preccurlyeq \widetilde{x}$.

Condition (8.4) is next used to establish two preliminary results on equilibrium, viz., that the price $p^{\star}$ is bounded and that the consumption $x_{h}^{\star}$ is therefore bounded away from zero. For this, the utility of each household $h$, is henceforth taken to have the form (8.1)-(8.2). The "instantaneous" utility a.k.a. felicity, $u_{h}$, is assumed to meet the following conditions, in addition to those of Lemma 8.1.

Continuity of Marginal Utility. For every $h$ and $m \in \mathbb{R}_{+}^{G}$

$$
\frac{\partial u_{h}}{\partial \mathrm{x}}(\cdot, \cdot, m) \in \mathcal{C}\left(T \times \mathbb{R}_{++}\right)
$$

i.e., the function $(t, \mathbf{x}) \mapsto\left(\partial u_{h} / \partial \mathbf{x}\right)(t, \mathbf{x}, m)$ is (jointly) continuous on $T \times \mathbb{R}_{++} .^{38}$

Boundedness of Marginal Utility (in $t$ ). For every $h$ and $(\mathrm{x}, m) \in \mathbb{R}_{++} \times \mathbb{R}_{+}^{G}$

$$
\sup _{t \in T} \frac{\partial u_{h}}{\partial \mathbf{x}}(t, \mathbf{x}, m)<+\infty \text {. }
$$

When $T$ is compact, this follows from the continuity of $\partial u_{h} / \partial \mathbf{x}$ in $t$.

Unboundedness of Marginal Utility (in x). For every $h$ and $m \in \mathbb{R}_{+}^{G}$

$$
\frac{\partial u_{h}}{\partial \mathbf{x}}(t, \mathbf{x}, m) \nearrow+\infty \quad \text { uniformly in } t \in T \text { as } \mathbf{x} \searrow 0 \text {. }
$$

Remark 8.3. Assume (8.13). If $x_{h}^{\mathrm{En}}=0$ for each $h$, then $y^{\star} \gg 0$.

Proof. By Lemma 8.1, $0 \ll \sum_{h} x_{h}^{\star}=y^{\star}$.

Proposition 8.4. In addition to (8.12) and (8.13), assume that:

1. The section $\mathbb{Y}\left(q^{\star}\right)$ of the total production set is a pseudo-symmetric subset of $L^{\infty}(T)$.

2. $x_{h}^{\mathrm{En}} \geq 0$ for each $h$.

\footnotetext{
${ }^{38}$ Partial continuity of $\partial u_{h} / \partial \mathrm{x}$ in $\mathrm{x}$ follows from its monotonicity, i.e., from the concavity (and differentiability) of $u_{h}$ in $\mathrm{x}$.
} 
3. $\left\langle p^{\star}, x_{h}^{\star}\right\rangle+r \cdot m_{h}^{\star}>0$ for each $h$, i.e., the equilibrium expenditures are positive.

4. $\operatorname{EssSup}\left(y^{\star}\right)>0$, i.e., the equilibrium net output of the differentiated good is nonzero (as is the case by Remark 8.3 if $x_{h}^{\mathrm{En}}=0$ for each $h$ ).

Then $p^{\star} \in L^{\infty}(T)$, i.e., the equilibrium price is essentially bounded.

Proof. The idea is that a sufficiently high price would depress the demand so much that it could not equal the supply, given that the output is then close to its peak by pseudo-symmetry.

Since $p^{\star} \geq 0$, it suffices to show that $p^{\star}$ is bounded from above. By (8.4), ${ }^{39}$ for each $h$ there exists a $\lambda_{h}^{*}>0$ such that, for a.e. $t \in T$,

$$
\lambda_{h}^{*} p(t)=\frac{\partial u_{h}}{\partial \mathrm{x}}\left(t, x_{h}^{*}(t), m_{h}^{*}\right) .
$$

Fix any positive numbers $\mathrm{y}$ and $\left(\mathrm{x}_{h}\right)$ with

$$
\sum_{h \in \text { Ho }} \mathrm{x}_{h}<\mathrm{y}<\operatorname{EssSup}\left(y^{\star}\right)
$$

and use (8.12) to define

$$
\mathrm{p}^{\prime}:=\max _{h \in \mathrm{Ho}} \sup _{t \in T} \frac{1}{\lambda_{h}^{*}} \frac{\partial u_{h}}{\partial \mathrm{x}}\left(t, \mathrm{x}_{h}\right)<+\infty
$$

with $m_{h}^{\star}$ suppressed from the notation henceforth. By (8.14),

$$
x_{h}^{\star}(t) \geq \mathrm{x}_{h} \Rightarrow p^{\star}(t)=\frac{1}{\lambda_{h}^{*}} \frac{\partial u_{h}}{\partial \mathrm{x}}\left(t, x_{h}^{\star}(t)\right) \leq \frac{1}{\lambda_{h}^{*}} \frac{\partial u_{h}}{\partial \mathrm{x}}\left(t, \mathrm{x}_{h}\right) \leq \mathrm{p}^{\prime} .
$$

Since $x_{h}^{\text {En }} \geq 0$, it follows that for a.e. $t$

$$
p^{\star}(t)>\mathbf{p}^{\prime} \Rightarrow y^{\star}(t) \leq \sum_{h} x_{h}^{\star}(t)<\sum_{h \in \text { Ho }} \mathrm{x}_{h}<\mathrm{y}<\operatorname{EssSup}\left(y^{\star}\right) .
$$

Since $\mathrm{y}<\operatorname{EssSup}\left(y^{\star}\right)$ and $\mathbb{Y}\left(q^{\star}\right)$ is pseudo-symmetric, there exists a $\mathrm{p}^{\prime \prime} \in \mathbb{R}$ such that, for a.e. $t$,

$$
p(t)>\mathbf{p}^{\prime \prime} \Rightarrow y(t) \geq \mathrm{y}
$$

Set $p=\max \left\{p^{\prime}, p^{\prime \prime}\right\}$. Then

$$
p^{\star}(t) \leq \mathrm{p} \quad \text { for almost every } t \in T
$$

because (8.15) and (8.16) contradict each other unless $\left\{t: p^{\star}(t)>\mathrm{p}\right\}$ is a null set.

Comments: When $\mathbb{Y}\left(q^{\star}\right)$ is symmetric, the Proof of Proposition 8.4 simplifies and strengthens:

\footnotetext{
${ }^{39}$ Lemma 8.1 applies because incomes (equal to expenditures) are positive.
} 
1. The number $p^{\prime}$ itself is a bound on $p^{\star}$. This is because, in terms of the sets

$$
A^{\prime}:=\left\{t \in T: p^{\star}(t)>\mathrm{p}^{\prime}\right\} \quad \text { and } \quad A^{\prime \prime}:=\left\{t \in T: y^{\star}(t) \geq \mathrm{y}\right\}
$$

the implication (8.15) means that

$$
\text { ess } \sup _{A^{\prime}} y^{\star}<\mathrm{y} \leq \operatorname{ess} \inf _{A^{\prime \prime}} y^{\star}
$$

and, since $y^{\star}$ and $p^{\star}$ are similarly arranged (Lemma 5.9), it follows that

$$
\operatorname{ess} \sup _{A^{\prime}} p^{\star} \leq \operatorname{ess} \inf _{A^{\prime \prime}} p^{\star} \leq \operatorname{ess} \sup _{A^{\prime \prime}} p^{\star} \leq \mathrm{p}^{\prime}
$$

(The penultimate inequality holds because $A^{\prime \prime}$ is $\sigma$-nonnull, whilst the last inequality holds by (8.15) again.) This shows that

$$
\operatorname{ess} \sup _{t: p^{\star}(t)>\mathrm{p}^{\prime}} p^{\star}(t) \leq \mathrm{p}^{\prime}
$$

which cannot be - unless $p^{\star} \leq \mathrm{p}^{\prime}$ a.e. (in which case $A^{\prime}$ is a null set and the supremum on $A^{\prime}$ is $-\infty$ ).

2. In the one-consumer case with $x^{\text {En }}=0$, the number $\mathrm{p}^{\prime}$ (which depends on $\mathrm{x}$ ) becomes the exact upper bound on $p^{\star}$ as $x / \operatorname{EssSup}\left(y^{\star}\right)$, since $\lambda^{\star} p^{\prime}$ decreases to

$$
\begin{aligned}
\inf _{\mathrm{x}: \mathrm{x}<\operatorname{Sup}\left(y^{\star}\right)} \sup _{t \in T} \frac{\partial u}{\partial \mathbf{x}}(t, \mathbf{x}) & =\sup _{t \in T} \inf _{\mathbf{x}: \mathbf{x}<\operatorname{Sup}\left(y^{\star}\right)} \frac{\partial u}{\partial \mathbf{x}}(t, \mathbf{x})=\sup _{t \in T} \frac{\partial u}{\partial \mathbf{x}}\left(t, \operatorname{EssSup}\left(y^{\star}\right)\right) \\
& =\lambda^{\star} \operatorname{EssSup}\left(p^{\star}\right) .
\end{aligned}
$$

Remark 8.3 can now be strengthened so that Example 6.2 can be applied to verify the sub-symmetry condition of Theorem 7.2.

Corollary 8.5. On the assumptions of Proposition 8.4, EssInf $\left(x_{h}^{\star}\right)>0$ for each $h$.

Proof. The idea is that a bounded price must mean a positive minimum consumption rate (since the marginal utility becomes infinite at zero).

By Proposition 8.4 and the uniformity of divergence in (8.13), for each $h$ there is a constant $\underline{\mathrm{x}}_{h}>0$ with

$$
\frac{\partial u_{h}}{\partial \mathbf{x}}\left(t, \underline{\mathbf{x}}_{h}\right)>\lambda_{h}^{\star} \operatorname{EssSup}\left(p^{\star}\right) \quad \text { for every } t \in T \text {. }
$$

It follows that

$$
x_{h}^{\star}(t)>\underline{x}_{h} \quad \text { for a.e. } t \in T
$$

since $x_{h}^{\star}(t) \leq \underline{x}_{h}$ implies that

$$
\frac{\partial u_{h}}{\partial \mathbf{x}}\left(t, x_{h}^{\star}(t)\right) \geq \frac{\partial u_{h}}{\partial \mathbf{x}}\left(t, \underline{x}_{h}\right)>\lambda_{h}^{\star} p^{\star}(t)
$$

which contradicts (8.14). 
Corollary 8.6. In addition to the assumptions of Proposition 8.4, assume (8.11). Then for each $h$ the set $S\left(x_{h}^{\star}, m_{h}^{\star}, \preccurlyeq h\right)$, defined by (6.3), is strongly sub-symmetric at $x_{h}^{\star}$.

Proof. This follows from Corollary 8.5 and Example 6.2.

With $\partial u_{h} / \partial \mathbf{x}$ infinite at $\mathbf{x}=0$ as per (8.13), continuity of the equilibrium price follows.

Corollary 8.7. In addition to (8.11), (8.12) and (8.13), assume (as in Theorem 7.2) that:

1. A price system $\left(p^{\star}, r^{\star}\right) \in L^{1}(T) \times \mathbb{R}^{G}$ supports a competitive equilibrium with a consumption allocation $\left(x_{h}^{\star}, m_{h}^{\star}\right)_{h \in \text { Ho }}$ and a total input-output bundle $\left(y^{\star}, q^{\star}\right) \in \mathbb{Y}$.

2. The section $\mathbb{Y}\left(q^{\star}\right)$ of the total production set is quasi-symmetric, and also pseudosymmetric.

3. $x_{h}^{\mathrm{En}} \in \mathcal{C}_{+}(T)$ for each $h$.

Assume also (as in Proposition 8.4) that:

4. $\left\langle p^{\star}, x_{h}^{\star}\right\rangle+r \cdot m_{h}^{\star}>0$ for each $h$.

5. $0<\operatorname{EssSup}\left(y^{\star}\right):=\operatorname{ess}_{\sup _{T}} y^{\star}$ (as is the case by Remark 8.3 when $x_{h}^{\mathrm{En}}=0$ for each $h$ ).

Then the equilibrium price has a continuous and bounded variant, viz., ess $p^{\star} \in$ $\mathcal{C}^{\mathrm{B}}(T) \cdot{ }^{40}$

Proof. This follows from Corollary 8.6 and Theorem 7.2.

When the equation $\lambda \mathrm{p}=\left(\partial u_{h} / \partial \mathbf{x}\right)(t, \mathbf{x})$ has a unique solution for $\mathbf{x}$, price continuity implies that the equilibrium quantities, $x_{h}^{\star}(t)$ and $y^{\star}(t)$, are also continuous in $t$.

Corollary 8.8. On the assumptions of Corollary 8.7, if additionally $T$ is compact and $\left(\partial u_{h} / \partial \mathbf{x}\right)(t, \cdot)$ is (strictly) decreasing, for each $t$-i.e., the "instantaneous" utility function $\mathrm{x} \mapsto u_{h}(t, \mathrm{x})$ is strictly concave (as well as differentiable on $\mathbb{R}_{++}$) -then the equilibrium consumption of the differentiated good has a continuous variant, viz., ess $x_{h}^{\star} \in \mathcal{C}(T)$.

Proof. As is shown below, $x_{h}^{\star}$ has a variant

$$
\breve{x}_{h}^{\star}: T \rightarrow\left[\underline{\mathrm{x}}_{h}, \overline{\mathrm{x}}_{h}\right]=\left[\operatorname{EssInf}\left(x_{h}^{\star}\right), \operatorname{EssSup}\left(x_{h}^{\star}\right)\right] \subset \mathbb{R}_{++}
$$

for which (8.14) holds everywhere on $T$, i.e.,

$$
\lambda_{h}^{*} \operatorname{ess} p^{\star}(t)=\frac{\partial u_{h}}{\partial \mathrm{x}}\left(t, \breve{x}_{h}^{\star}(t)\right) \quad \text { for every } t \in T .
$$

(To see this, start from any variant $\breve{x}_{h}^{\star}: T \rightarrow\left[\underline{x}_{h}, \bar{x}_{h}\right]$, for which (8.18) holds for $t$ outside of some $\sigma$-null set $Z$. For any $t \in Z$ choose a sequence $t_{n} \in T \backslash Z$ with $t_{n} \rightarrow t$ as $n \rightarrow+\infty$, then choose a limit point $\mathrm{x}$ of the sequence $\left(\breve{x}_{h}^{\star}\left(t_{n}\right)\right)$, and redefine $\breve{x}_{h}^{\star}(t)$ as $\mathrm{x}$. Since (8.18) holds along the sequence $t_{n}$, it also holds at $t=\lim _{n} t_{n}$ because $\partial u_{h} / \partial \mathbf{x}$ and ess $p^{\star}$ are continuous by (8.11) and Corollary 8.7.)

\footnotetext{
${ }^{40}$ Because of $(8.12), p^{\star}$ is bounded even if $T$ is not compact.
} 
Since $u_{h}(t, \cdot)$ is strictly concave, $(8.18)$ can be inverted to give $\breve{x}_{h}^{\star}$ as the composition

$$
\breve{x}_{h}^{\star}(t)=\left(\frac{\partial u_{h}}{\partial \mathrm{x}}(t, \cdot)\right)^{-1}\left(\lambda_{h}^{*} \operatorname{ess} p^{\star}(t)\right) \quad \text { for every } t \in T \text {. }
$$

By (8.11), Lemma B.3 applies to $M=\partial u_{h} / \partial \mathrm{x}$ with $K=\left[\underline{\mathrm{x}}_{h}, \overline{\mathrm{x}}_{h}\right]$; and it shows that the function

$$
(t, \mathbf{r}) \mapsto\left(\frac{\partial u_{h}}{\partial \mathrm{x}}(t, \cdot)\right)^{-1}(\mathrm{r})
$$

is continuous (in $t$ and $r$ jointly). Since ess $p^{\star}$ is continuous on $T$ by Corollary 8.7, it follows from (8.19) that so is $\breve{x}_{h}^{\star}$ (and also that $\breve{x}_{h}^{\star}=\operatorname{ess} x_{h}^{\star}$ ).

\section{Application to peak-LOAd PRicing With Storage}

The price continuity theorem is next applied to electricity pricing with (or without) pumped storage. This extends the results of Sections 2 and 3 to the case of cross-price dependent demand, provided that the preferences and technologies of electricity users meet the weak "symmetry-like" conditions. To present this application rigorously yet briefly, we assume that:

1. As a result of aggregating commodities on the basis of some fixed relative prices, there are just two consumption goods apart from electricity - viz., a numeraire (measured in \$) and a homogeneous final good produced with an input of electricity.

2. The various kinds of thermal generating capacity and fuel have fixed prices, $r_{\mathrm{Th}}=$ $\left(r_{1}, \ldots, r_{\Theta}\right)$ and $w=\left(w_{1}, \ldots, w_{\Theta}\right)$, in terms of the numeraire (i.e., in $\$ / \mathrm{kW}$ and in $\$ / \mathrm{kWh}$, respectively). The prices of storage and conversion capacities, $r_{\mathrm{PS}}=\left(r_{\mathrm{St}}, r_{\mathrm{Co}}\right)$, are also fixed (in $\$ / \mathrm{kWh}$ and in $\$ / \mathrm{kW}$ ).

A complete commodity bundle consists therefore of electricity (a differentiated good) and of a number of homogeneous goods, viz., the thermal capacities, the fuels, the storage and conversion capacities, the produced final good and the numeraire. The quantities are always written in this order; but those which are irrelevant in a particular context (and can be set equal to zero) may be omitted for brevity. For example, a consumption bundle consists of electricity, the produced final good and the numeraire; so it may be written as $(x ; \varphi, m) \in L^{\infty}[0, T] \times \mathbb{R}^{2}$. A matching consumer price system is $(p ; \varrho, 1) \in L^{\infty *}[0, T] \times \mathbb{R}^{2}$ (whilst a complete price system is $\left.\left(p ; r_{\mathrm{Th}} ; w ; r_{\mathrm{PS}} ; \varrho, 1\right)\right)$. There is a finite set, Ho, of households; and for each $h \in$ Ho the utility function $U_{h}$ is $\mathrm{m}\left(L^{\infty} \times \mathbb{R}^{2}, L^{1} \times \mathbb{R}^{2}\right)$-continuous (i.e., Mackey continuous) on the consumption set $L_{+}^{\infty}[0, T] \times \mathbb{R}_{+}^{2}$. Each household's initial endowment is a quantity $m_{h}^{\text {En }}>0$ of the numeraire only; and nonsatiation in this commodity is assumed. The preference order can of course be regarded as defined on the orthant in the full commodity space $L:=L^{\infty}[0, T] \times \mathbb{R}^{2 \Theta+4}$ by positing that the consumer has no use for the electricity supplier's inputs, which are $k_{\mathrm{Th}}=\left(k_{1}, \ldots, k_{\Theta}\right), v=\left(v_{1}, \ldots, v_{\Theta}\right)$ 
and $k_{\mathrm{PS}}=\left(k_{\mathrm{St}}, k_{\mathrm{Co}}\right)$. This means regarding a utility $U_{h}$ on $L_{+}^{\infty} \times \mathbb{R}_{+}^{2}$ as a function on $L_{+}^{\infty} \times \mathbb{R}_{+}^{2 \Theta+2+2}$ defined by $\left(x ; k_{\mathrm{Th}}, v ; k_{\mathrm{PS}} ; \varphi, m\right) \mapsto U_{h}(x ; \varphi, m)$.

There is also an industrial user, producing the final good from inputs of electricity and the numeraire. (In the case of decreasing returns to scale, each household's share $\varsigma_{h}$ in the user industry's profits must be specified.) The user's production function $F: L_{+}^{\infty}[0, T] \times \mathbb{R}_{+} \rightarrow \mathbb{R}$ is also assumed to be concave, nondecreasing and $\mathrm{m}\left(L^{\infty} \times \mathbb{R}, L^{1} \times \mathbb{R}\right)$-continuous, and to have a nonzero recession function; i.e., the user's production set $\mathbb{Y}_{\mathrm{IU}}$ contains an activity with constant returns to scale, other than free disposal. The section of $\mathbb{Y}_{\mathrm{IU}}$ through any $(\varphi,-m) \in \mathbb{R} \times \mathbb{R}_{-}$, which is

$$
\mathbb{Y}_{\mathrm{IU}}(\varphi,-m)=\left\{-z \in L_{-}^{\infty}[0, T]: F(z, m) \geq \varphi\right\}
$$

is assumed to be sub-symmetric.

The electricity supplier uses a multi-station thermal technology and pumped storage. Each thermal technique $\theta$ generates an output flow $y_{\theta}$ from the inputs of fuel $v_{\theta}$ (in $\mathrm{kWh}$ ), and of generating capacity $k_{\theta}$ (measured in $\mathrm{kW}$, like the output rate $\left.y_{\theta}(t)\right)$. The LR production set of technique $\theta=1, \ldots, \Theta$ is the cone

$$
\mathbb{Y}_{\theta}:=\left\{\left(y_{\theta} ;-k_{\theta},-v_{\theta}\right) \in L^{\infty}[0, T] \times \mathbb{R}_{-}^{2}: \operatorname{EssSup}\left(y_{\theta}^{+}\right) \leq k_{\theta}, \int_{0}^{T} y_{\theta}^{+} \mathrm{d} t \leq v_{\theta}\right\}
$$

and the total thermal production set is

$$
\mathbb{Y}_{\mathrm{Th}}:=\left\{\left(\sum_{\theta=1}^{\Theta} y_{\theta} ;-\left(k_{\theta}, v_{\theta}\right)_{\theta \in \Theta}\right):\left(y_{\theta} ;-k_{\theta},-v_{\theta}\right) \in \mathbb{Y}_{\theta} \text { for } \theta=1, \ldots, \Theta\right\}
$$

This is the sum of the $\mathbb{Y}_{\theta}$ 's, each of which is embedded in $L^{\infty} \times\left(\mathbb{R}_{-}^{2}\right)^{\Theta}$; different types of station are assumed to use different fuels, for simplicity. The set $\mathbb{Y}_{\mathrm{Th}}$ is further embedded in $L=\left(L^{\infty} \times \mathbb{R}^{2 \Theta}\right) \times \mathbb{R}^{4}$ as $\mathbb{Y}_{\mathrm{Th}} \times\{(0, \ldots, 0)\}$. The other production sets are also embedded in $L$ by inserting zeros at the appropriate positions.

To justify formally the fixed prices of inputs for electricity supply $\left(r_{\mathrm{Th}}, w\right.$ and $\left.r_{\mathrm{PS}}\right)$, there is the production set with the locus equation $r \cdot k+w \cdot v+m=0$ in the $(k, v, m)$-space.

Thermal generation is supplemented by pumped storage. In this technique, the signed outflow of energy from the reservoir, $-\dot{s}(t)=-\mathrm{d} s / \mathrm{d} t$, is a bounded function of time $t \in[0, T]$. Energy is moved in and out of storage with a converter, which is taken to be perfectly efficient and symmetrically reversible: this means that in a unit time a unit converter can either turn a unit of the marketed good (electricity) into a unit of the stocked intermediate good (a storable form of energy), or vice versa. On this simplifying assumption, $-\dot{s}(t)$ equals the rate of net flow from storage, $y_{\mathrm{PS}}(t)=\left(y_{\mathrm{PS}}^{+}-y_{\mathrm{PS}}^{-}\right)(t)$. The converter's capacity is denoted by $k_{\mathrm{Co}}$ (measured in $\mathrm{kW}$ ). The reservoir's capacity is $k_{\mathrm{St}}$ (in $\mathrm{kWh}$ ); stock can be held in storage at no 
running cost (or loss of stock). The LR production set is, therefore,

$$
\begin{aligned}
& \mathbb{Y}_{\mathrm{PS}}:=\left\{\left(y ;-k_{\mathrm{St}},-k_{\mathrm{Co}}\right) \in L^{\infty}[0, T] \times \mathbb{R}_{-}^{2}:|y| \leq k_{\mathrm{Co}},\right. \\
&\text { and } \left.\exists s \dot{s}=-y, s(0)=s(T), \text { and } 0 \leq s \leq k_{\mathrm{St}}\right\} .
\end{aligned}
$$

The minimum requirements for storage capacity and conversion capacity, when the (signed) output from storage is $y_{\mathrm{PS}}$ with

$$
\begin{aligned}
& \check{k}_{\mathrm{St}}\left(y_{\mathrm{PS}}\right)=\max _{t \in[0, T]} \int_{0}^{t} y_{\mathrm{PS}}(t) \mathrm{d} t+\max _{t \in[0, T]} \int_{t}^{T} y_{\mathrm{PS}}(t) \mathrm{d} t \\
& \check{k}_{\mathrm{Co}}\left(y_{\mathrm{PS}}\right)=\left\|y_{\mathrm{PS}}\right\|_{\infty}=\mathrm{ess} \sup _{t \in[0, T]}\left|y_{\mathrm{PS}}(t)\right| .
\end{aligned}
$$

(Formula (9.3) is derived in [11].) In these terms, $\left(y_{\mathrm{PS}},-k_{\mathrm{St}},-k_{\mathrm{Co}}\right) \in \mathbb{Y}_{\mathrm{PS}}$ if and only if

$$
\int_{0}^{T} y_{\mathrm{PS}}(t) \mathrm{d} t=0, \check{k}_{\mathrm{St}}\left(y_{\mathrm{PS}}\right) \leq k_{\mathrm{St}} \text { and } \check{k}_{\mathrm{Co}}\left(y_{\mathrm{PS}}\right) \leq k_{\mathrm{Co}}
$$

Unlike the thermal capacity and fuel requirements in (9.2), and unlike $\check{k}_{\text {Co }}(y)$, the storage capacity requirement $\breve{k}_{\mathrm{St}}(y)$ is not a symmetric function of $y$. But the storage technology does meet the quasi-symmetry condition. We verify this by formalising the following heuristic, in which $\psi$ is the price of stock: take an electricity price $p$ which jumps at some $t$, i.e., $p(t-)<p(t+)$. Can the supply (from storage plant) drop? If it does, i.e., $y(t-)>y(t+)$, then obviously $y(t-)>-k_{\mathrm{Co}}$ and $y(t+)<k_{\mathrm{Co}}$, so $p(t-) \geq \psi(t-)$ and $p(t+) \leq \psi(t+)$. Hence $0<p(t+)-p(t-) \leq \psi(t+)-\psi(t-)$, so the reservoir must be full at $t$, and it cannot be being discharged just before $t$ or charged just after $t$; i.e., $y(t-) \leq 0 \leq y(t+)$. This contradicts the drop in $y$. But the argument is incomplete because $p$ and $y$ may fail to have the one-sided limits. The need to make it rigorous is what has led us to the concept of quasi-symmetry.

Lemma 9.1. For every $k_{\mathrm{PS}}=\left(k_{\mathrm{St}}, k_{\mathrm{Co}}\right) \in \mathbb{R}_{+}^{2}$, the set of feasible flows from storage, $\mathbb{Y}_{\mathrm{PS}}\left(-k_{\mathrm{PS}}\right) \subset L^{\infty}[0, T]$, is quasi-symmetric (with the usual topology on $[0, T]$ ).

Proof. Given a $p \in L^{1}[0, T]$ and any $t_{\ddagger} \in[0, T] \backslash$ dom ess $p$, take a storage policy $y$ that maximises the operating profit $\langle p, \cdot\rangle$ on $\mathbb{Y}_{\mathrm{PS}}\left(-k_{\mathrm{PS}}\right)$. If $k_{\mathrm{St}}=0$ or $k_{\mathrm{Co}}=0$, then $y=0$ and there is nothing to prove (since (5.21) holds by Remark 5.15). So assume that $k_{\mathrm{St}}>0$ and $k_{\mathrm{Co}}>0$. The stock trajectory associated with $y$ is

$$
s(t)=-\int_{0}^{t} y(\tau) \mathrm{d} \tau+\max _{t \in[0, T]} \int_{0}^{t} y(\tau) \mathrm{d} \tau
$$

(since the second summand is the initial stock required for $s(t)$ never to fall below $0)$. The sets of those times when the reservoir is empty or full or neither are

$$
\begin{aligned}
& E:=\{t \in[0, T]: s(t)=0\} \\
& F:=\left\{t \in[0, T]: s(t)=k_{\mathrm{St}}\right\} .
\end{aligned}
$$


As we show in [11] and [16], there exists a function $\psi$ - which is the marginal value of stock as a function of time (and depends of course on $p$ and $k$ ) - with the following properties:

1. $\psi$ is of bounded variation on the interval $(0, T)$, with $\psi(0):=\psi(0+)$ and $\psi(T):=\psi(T-)$ by convention.

2. $\psi$ rises only on $F$ and falls only on $E$. In formal terms, the sets $F$ and $E$ support, respectively, the nonnegative and nonpositive parts of the signed Borel measure defined by

$$
\mathrm{d} \psi\left[t^{\prime}, t^{\prime \prime}\right]:=\psi\left(t^{\prime \prime}+\right)-\psi\left(t^{\prime}-\right)
$$

for $t^{\prime} \leq t^{\prime \prime}$, with $\psi(0-)=\psi(T+)$ set equal to any number between $\psi(0+)$ and $\psi(T-) \cdot{ }^{41}$ In symbols, $\operatorname{supp}(\mathrm{d} \psi)^{+} \subseteq F$ and $\operatorname{supp}(\mathrm{d} \psi)^{-} \subseteq E$.

3. The optimum output is of the "bang-bang" type on the set $\{t: p(t) \neq \psi(t)\}$; i.e., for a.e. $t \in[0, T]$

$$
y(t)=\left\{\begin{array}{ll}
k_{\mathrm{Co}} & \text { if } p(t)>\psi(t) \\
-k_{\mathrm{Co}} & \text { if } p(t)<\psi(t)
\end{array} .\right.
$$

For simplicity choose variants of $p$ and $y$ which satisfy (9.10) for every $t$. There are two cases, which require different arguments: "from prices to quantities" if $\psi$ is continuous, and "from quantities to prices" if $\psi$ is discontinuous. ${ }^{42}$

Case 1: If $\psi$ is continuous at $t_{\ddagger}$, take any $\alpha>0$ that is less than $\bar{p}\left(t_{\ddagger}\right)-\underline{p}\left(t_{\ddagger}\right)$ if $\bar{p}\left(t_{\ddagger}\right)$ and $p\left(t_{\ddagger}\right)$ are finite. Fix any positive number $\beta \leq \alpha$ and an $I \in \mathcal{N}\left(\overline{t_{\ddagger}}\right)$ such that $\left|\psi(t)-\psi\left(t_{\ddagger}\right)\right|<\beta / 2$ for every $t \in I$. By Remark 5.15, for every $N \in \mathcal{N}\left(t_{\ddagger}\right)$ there exist sets $A^{\prime} \subset N \cap I$ and $A^{\prime \prime} \subset N \cap I$, both of positive measure, with

$$
\alpha+p\left(t^{\prime}\right) \leq p\left(t^{\prime \prime}\right)
$$

for every $t^{\prime} \in A^{\prime}$ and $t^{\prime \prime} \in A^{\prime \prime}$. Suppose that $y\left(t^{\prime}\right)>y\left(t^{\prime \prime}\right)$ for some $t^{\prime} \in A^{\prime}$ and $t^{\prime \prime} \in A^{\prime \prime}$; then of course $y\left(t^{\prime}\right)>-k_{\mathrm{Co}}$ and $y\left(t^{\prime \prime}\right)<k_{\mathrm{Co}}$, so $p\left(t^{\prime}\right) \geq \psi\left(t^{\prime}\right)$ and $p\left(t^{\prime \prime}\right) \leq \psi\left(t^{\prime \prime}\right)$. Hence the contradiction

$$
\alpha \leq p\left(t^{\prime \prime}\right)-p\left(t^{\prime}\right) \leq \psi\left(t^{\prime \prime}\right)-\psi\left(t^{\prime}\right)<\beta \leq \alpha,
$$

which shows that actually $y\left(t^{\prime}\right) \leq y\left(t^{\prime \prime}\right)$ for every $t^{\prime} \in A^{\prime}$ and $t^{\prime \prime} \in A^{\prime \prime}$. (This case includes the cases of $t_{\ddagger}=0$ and $t_{\ddagger}=T$, since at the endpoints $\psi$ is defined by continuity, which means one-sided continuity.)

Case 2: If $\psi$ is discontinuous, at a $t_{\ddagger} \in(0, T)$, then $\psi\left(t_{\ddagger}-\right) \neq \psi\left(t_{\ddagger}+\right)$, so $t_{\ddagger} \in E \cup F$. Consider, e.g., the case of $\psi\left(t_{\ddagger}-\right)<\psi\left(t_{\ddagger}+\right)$, in which $t_{\ddagger} \in F$. Take any positive

$$
\alpha<\psi\left(t_{\ddagger}+\right)-\psi\left(t_{\ddagger}-\right)
$$

\footnotetext{
${ }^{41}$ So $\mathrm{d} \psi$ has zero total mass, and $\mathrm{d} \psi\{0\}$ and $\mathrm{d} \psi\{T\}$ do not have opposite signs (which is convenient when 0 and $T$ are viewed as one point of a circle).

${ }^{42}$ Case 2 does not arise if $p$ is continuous [11], but here the continuity of $p$ must not be assumed (since it is, of course, to be proved by using the present lemma).
} 
and any positive $\epsilon \leq \psi\left(t_{\ddagger}+\right)-\psi\left(t_{\ddagger}-\right)-\alpha$; and fix an $I \in \mathcal{N}\left(t_{\ddagger}\right)$ such that $\left|\psi(t)-\psi\left(t_{\ddagger}-\right)\right| \leq \epsilon / 2$ for every $t \in I \cap\left(0, t_{\ddagger}\right)$ and $\left|\psi(t)-\psi\left(t_{\ddagger}+\right)\right| \leq \epsilon / 2$ for every $t \in I \cap\left(t_{\ddagger}, T\right)$. Being full at $t_{\ddagger}$, the reservoir cannot be being discharged just before $t_{\ddagger}$ or charged just after $t_{\ddagger}$. In formal terms, for every $N \in \mathcal{N}\left(t_{\ddagger}\right)$ it cannot be that $y>0$ a.e. on $N \cap I \cap\left(0, t_{\ddagger}\right)$; i.e., $y \leq 0$ on some nonnull set $A^{\prime} \subseteq N \cap I \cap\left(0, t_{\ddagger}\right)$. $A$ fortiori $p \leq \psi$ on $A^{\prime}$. Similarly, on some nonnull set $A^{\prime \prime} \subseteq N \cap I \cap\left(t_{\ddagger}, T\right)$ one has $y \geq 0$ and, hence, $p \geq \psi$ on $A^{\prime \prime}$. So, for every $t^{\prime} \in A^{\prime} \subseteq N$ and $t^{\prime \prime} \in A^{\prime \prime} \subseteq N$,

$$
p\left(t^{\prime}\right) \leq \psi\left(t^{\prime}\right) \leq \psi\left(t_{\ddagger}-\right)+\frac{\epsilon}{2} \quad \text { and } \quad p\left(t^{\prime \prime}\right) \geq \psi\left(t^{\prime \prime}\right) \geq \psi\left(t_{\ddagger}+\right)-\frac{\epsilon}{2}
$$

and therefore

$$
p\left(t^{\prime \prime}\right)-p\left(t^{\prime}\right) \geq \psi\left(t_{\ddagger}+\right)-\psi\left(t_{\ddagger}-\right)-\epsilon \geq \alpha .
$$

This completes the proof, since

$$
y\left(t^{\prime \prime}\right) \geq 0 \geq y\left(t^{\prime}\right)
$$

by the very choice of $A^{\prime}$ and $A^{\prime \prime}$. (The case of $\psi\left(t_{\ddagger}-\right)>\psi\left(t_{\ddagger}+\right)$, in which $t_{\ddagger} \in E$, is handled in a similar way.)

Comment: For Case 2 the Proof of Lemma 9.1 shows also that, for $t_{\ddagger} \in F$,

$$
\begin{aligned}
& \psi\left(t_{\ddagger}-\right) \geq \operatorname{ess} \liminf _{\tau \nearrow t_{\ddagger}} p(\tau) \geq \operatorname{ess} \liminf _{\tau \rightarrow t_{\ddagger}} p(\tau)=\underline{p}\left(t_{\ddagger}\right) \\
& \psi\left(t_{\ddagger}+\right) \leq \operatorname{ess} \limsup _{\tau \searrow t_{\ddagger}} p(\tau) \leq \operatorname{ess} \limsup _{\tau \rightarrow t_{\ddagger}} p(\tau)=\bar{p}\left(t_{\ddagger}\right)
\end{aligned}
$$

and so

$$
0 \leq \psi\left(t_{\ddagger}+\right)-\psi\left(t_{\ddagger}-\right) \leq \bar{p}\left(t_{\ddagger}\right)-\underline{p}\left(t_{\ddagger}\right) .
$$

When the last inequality is strict, the choice of $\alpha$ for Case 2 can be improved: as in Case 1, any

$$
\alpha<\bar{p}\left(t_{\ddagger}\right)-\underline{p}\left(t_{\ddagger}\right)
$$

will do. For $\alpha>\psi\left(t_{\ddagger}+\right)-\psi\left(t_{\ddagger}-\right)$ this is shown as in Case 1: take any positive $\beta \leq \alpha-\left(\psi\left(t_{\ddagger}+\right)-\psi\left(t_{\ddagger}-\right)\right)$, and fix an $I \in \mathcal{N}\left(t_{\ddagger}\right)$ such that $\psi(t)>\psi\left(t_{\ddagger}-\right)-\beta / 2$ and $\psi(t)<\psi\left(t_{\ddagger}+\right)+\beta / 2$ for every $t \in I$. Again by Remark 5.15 , for every $N \in \mathcal{N}\left(t_{\ddagger}\right)$ there exist nonnull sets $A^{\prime} \subset N \cap I$ and $A^{\prime \prime} \subset N \cap I$ with

$$
\alpha+p\left(t^{\prime}\right) \leq p\left(t^{\prime \prime}\right)
$$

for every $t^{\prime} \in A^{\prime}$ and $t^{\prime \prime} \in A^{\prime \prime}$. Suppose contrarily that $y\left(t^{\prime}\right)>y\left(t^{\prime \prime}\right)$ for some $t^{\prime} \in A^{\prime}$ and $t^{\prime \prime} \in A^{\prime \prime}$; then $y\left(t^{\prime}\right)>-k_{\mathrm{Co}}$ and $y\left(t^{\prime \prime}\right)<k_{\mathrm{Co}}$, so $p\left(t^{\prime}\right) \geq \psi\left(t^{\prime}\right)$ and $p\left(t^{\prime \prime}\right) \leq \psi\left(t^{\prime \prime}\right)$. Hence the contradiction

$$
\alpha \leq p\left(t^{\prime \prime}\right)-p\left(t^{\prime}\right) \leq \psi\left(t^{\prime \prime}\right)-\psi\left(t^{\prime}\right)<\psi\left(t_{\ddagger}+\right)-\psi\left(t_{\ddagger}-\right)+\beta \leq \alpha
$$

which shows, for every $t^{\prime} \in A^{\prime}$ and $t^{\prime \prime} \in A^{\prime \prime}$, that actually $y\left(t^{\prime}\right) \leq y\left(t^{\prime \prime}\right)$.

The Mackey continuity assumptions on the users' utility and production functions, $U_{h}$ and $F$, mean that electricity consumption is interruptible (i.e., a brief interruption 
causes only a small loss of utility or output). As we show in [12], this guarantees that the equilibrium TOU price is a density, i.e., a time-dependent rate in $\$ / \mathrm{kWh}$. Subsymmetry conditions on $U_{h}$ and $F$ guarantee that the price density is continuous.

Theorem 9.2. The electricity pricing model has a long-run competitive equilibrium. Furthermore:

1. If an equilibrium tariff $p^{\star} \in L_{+}^{\infty *}[0, T]$ supports (together with some price $\varrho^{\star} \in$ $\mathbb{R}_{+}$for the other produced good) an equilibrium allocation with a nonzero electricity output $y_{\mathrm{Th}}^{\star}+y_{\mathrm{PS}}^{\star}$ (from thermal generation and pumped storage), then $p^{\star} \in L_{+}^{1}[0, T]$.

2. If additionally the set $\left\{x \in L_{+}^{\infty}: U_{h}\left(x, \varphi_{h}^{\star}, m_{h}^{\star}\right) \geq U_{h}\left(x_{h}^{\star}, \varphi_{h}^{\star}, m_{h}^{\star}\right)\right\}$ is strongly sub-symmetric at $x_{h}^{\star}$ (for each household $h$ ), then $p^{\star}$ has a continuous variant, viz., ess $p^{\star} \in \mathcal{C}[0, T]$.

Proof. By [1, Theorem 1] there is an equilibrium price system with $p^{\star} \in L_{+}^{\infty *}$. And actually $p^{\star} \in L^{1}$, as we show in [12]. This proves Part 1 .

For Part 2, we verify the assumptions of Theorem 7.2. Since each of the $\mathbb{Y}_{\theta}$ 's has symmetric sections, so does their sum $\mathbb{Y}_{\mathrm{Th}}$ (Lemma 5.20). Since $\mathbb{Y}_{\mathrm{PS}}$ has quasisymmetric sections (Lemma 9.1), and $\mathbb{Y}_{I U}$ has sub-symmetric sections (by assumption), the total production set has quasi-symmetric sections (Lemma 5.21). For households, strong sub-symmetry at $x_{h}^{\star}$ is assumed. And $x_{h}^{\mathrm{En}}=0 \in \mathcal{C}_{+}[0, T]$ trivially.

The conditions on $U_{h}$ and $F$ are met by additively separable utility and production functions with a continuous marginal utility or productivity (Sections 5, 6 and 8). This case uses Proposition 8.4, and hence it requires the following result.

Lemma 9.3. For each $k_{\mathrm{PS}}=\left(k_{\mathrm{St}}, k_{\mathrm{Co}}\right) \in \mathbb{R}_{+}^{2}$, the set $\mathbb{Y}_{\mathrm{PS}}\left(-k_{\mathrm{PS}}\right)$ is pseudo-symmetric.

Proof. The function $\psi$ on $[0, T]$, introduced in the Proof of Lemma 9.1, is bounded (since it is of bounded variation). By (9.10), if $p(t)>\operatorname{Sup}(\psi)$ then $y(t)=k_{\mathrm{Co}} \geq$ $\operatorname{EssSup}(y)$. So (5.25) holds even with $\delta=0$ (and with $\mathrm{p}=\operatorname{Sup}(\psi)$ ).

Corollary 9.4. Assume that:

1. As in Section 8, each household's utility $U_{h}$ has the (concave) integral form (8.2), with the instantaneous utility from electricity, $u_{h}(\cdot, \cdot, \varphi, m)$, satisfying (8.11), (8.12) and (8.13) for any $(\varphi, m) \in \mathbb{R}_{+}^{2}$ (in place of $m \in \mathbb{R}_{+}^{G}$ ).

2. The industrial user's production function, $F$, has the integral form (5.17).

Then any equilibrium tariff has a continuous variant, viz., ess $p^{\star} \in \mathcal{C}_{++}[0, T]$.

For each $h$, if $u_{h}$ is strictly concave (in its second variable, $\mathrm{x}$ ), then the equilibrium consumption of electricity has a continuous variant, ess $x_{h}^{\star} \in \mathcal{C}_{++}[0, T]$.

Proof. To show that $p^{\star}$ is continuous, we verify the assumptions of Corollary 8.7. As in the Proof of Theorem 9.2, the set $\mathbb{Y}_{\mathrm{Th}}$ has symmetric sections and $\mathbb{Y}_{\mathrm{PS}}$ has quasisymmetric sections. Additionally $\mathbb{Y}_{\mathrm{IU}}$ has sub-symmetric sections (Example 5.13), so the total production set has quasi-symmetric sections (by Parts 1 and 2 of Lemma 5.21). 
Also, both $\mathbb{Y}_{\mathrm{PS}}$ and $\mathbb{Y}_{\mathrm{IU}}$ have pseudo-symmetric sections (Lemma 9.3 and Example 5.19); so the total production set has pseudo-symmetric sections (by Part 3 of Lemma 5.21). Finally, $x_{h}^{\mathrm{En}}=0$ for each $h$ by assumption (so $y_{\mathrm{Th}}^{\star}+y_{\mathrm{PS}}^{\star}-z^{\star}$, the electricity output net of industrial consumption, is positive by Remark 8.3).

By assumption $u_{h}$ is increasing (in $\mathrm{x}$ ), so $p^{\star} \gg 0$ by (8.4). Also, with $u_{h}$ strictly concave (in $\mathrm{x}$ ), $x_{h}^{\star}$ is continuous by Corollary 8.8. And $\operatorname{Min}\left(x_{h}^{\star}\right)>0$ by Corollary 8.5.

\section{Conclusions}

Equilibrium pricing of a continuous-time flow such as electricity is likely to require a continuously varying price, to eliminate the demand jumps caused by sudden switches between different price rates. Price continuity is also important for other reasons, for example in rental valuation of storage plants. With cross-price independent demand and supply curves, an equilibrium price varies continuously if the curves do. A more general result is based on ideas from the Hardy-Littlewood-Pólya theory of rearrangements. In particular, cost symmetry implies "similar arrangement" of price and output trajectories. The assumption can be weakened for use with "nonsymmetric" technologies (such as energy storage), and it can also be adapted for use with preferences. This gives what we believe to be the first applicable price continuity result for competitive equilibrium in Lebesgue commodity and price spaces $L^{\rho}(T)$, i.e., the spaces of bounded or integrable functions on a (topological) measure space of commodity characteristics.

\section{Appendix A. Lower And upper essential Values}

Assume that $T$ is a (Hausdorff) topological space, and that $\sigma$ is a measure on a sigma-algebra, $\mathcal{A}$, of subsets of $T$ that contains the Borel sigma-algebra. Recall that $L^{0}(T, \sigma)$ is the vector space of all ( $\sigma$-equivalence classes of) measurable $\mathbb{R}$-valued functions on $T$.

Definition A.1. For every $p \in L^{0}(T)$, the lower essential value of $p$ at $t$ is

$$
\underline{\operatorname{ess}} p(t):=\sup _{N \in \mathcal{N}(t)} \operatorname{ess} \inf _{N} p
$$

i.e., it is the supremum, on the neighbourhood system $\mathcal{N}(t)$, of the essential infimum of $p$ in any neighbourhood, $N$, of $t$. The upper essential value of $p$ at $t$ is

$$
\overline{\operatorname{ess}} p(t):=\inf _{N \in \mathcal{N}(t)} \operatorname{ess} \sup _{N} p .
$$

The notations are abbreviated to $\underline{p}$ and $\bar{p}$. Where $\underline{p}(t)$ and $\bar{p}(t)$ are equal and finite, their common value is the essential value of $p$ at $t$, denoted by ess $p(t)$. Its domain is

$$
\operatorname{dom} \operatorname{ess} p=\{t \in T:-\infty<\underline{p}(t)=\bar{p}(t)<+\infty\} \text {. }
$$

Comments:

1. The essential values are literally functions (rather than equivalence classes). 
2. For $p \in L^{1}$, equivalent definitions of $\underline{p}, \bar{p}$ and ess $p$ are given in, e.g., [29, II.9: pp. 89-90].

3. $\operatorname{ess} \inf _{T} p \leq \underline{p}(t)$ and $\bar{p}(t) \leq \operatorname{ess}_{\sup _{T}} p$, with equalities at some $t \in T$. So unless $p \in L^{\infty}$, both $\underline{p}$ and $\bar{p}$ are extended real-valued functions, from $T$ into $\mathbb{R} \cup\{ \pm \infty\}$. But ess $p$ is finite (on dom ess $p$ ).

4. The lower and upper essential limits of $p$ at $t$ are usually defined by means of pierced neighbourhoods, i.e.,

$$
\begin{aligned}
\operatorname{ess} \liminf _{\tau \rightarrow t} p(\tau) & :=\sup _{N \in \mathcal{N}(t)} \text { ess } \inf _{N \backslash\{t\}} p \\
\text { ess } \limsup _{\tau \rightarrow t} p(\tau) & :=\inf _{N \in \mathcal{N}(t)} \text { ess } \sup _{N \backslash\{t\}} p .
\end{aligned}
$$

The limits are identical to the values (A.1)-(A.2) if $\sigma\{t\}=0$. This is the case in [5, IV.36-IV.37] - where $\sigma=$ meas, and $T$ is an interval of $\mathbb{R}$. The one-sided, left or right, essential limits are then defined as well.

5. If $\sigma\{t\}>0$ (i.e., $t$ is an atom for $\sigma$ ) then

$$
\begin{aligned}
& \underline{\operatorname{ess}} p(t)=\min \left\{p(t), \text { ess } \liminf _{\tau \rightarrow t} p(\tau)\right\} \\
& \overline{\operatorname{ess}} p(t)=\max \left\{p(t), \operatorname{ess} \limsup _{\tau \rightarrow t} p(\tau)\right\} .
\end{aligned}
$$

6. The essential limit is the limit for the essential topology [5, p. 105].

The following account of the key results on essential values combines those of $[5$, IV.37] and [29, II.9: pp. 90, 91, 94].

Lemma A.2. For every $p \in L^{0}(T, \sigma)$ :

1. The lower value $\underline{p}$ is lower semicontinuous, and the upper value $\bar{p}$ is upper semicontinuous (each as a function from $T$ into $\mathbb{R} \cup\{ \pm \infty\}$ ).

2. If the topology of $T$ has a countable base of open sets, then $\underline{p} \leq p \leq \bar{p}$ almost everywhere on $T$ (w.r.t. $\sigma$ ).

3. If every nonempty open subset of $T$ is $\sigma$-nonnull, ${ }^{43}$ then $\underline{p} \leq \bar{p}$ everywhere on $T$.

Proof. For Part 1, fix any $t \in T$ and any number $\mathrm{p}>\bar{p}(t)$. By the definition (A.2) of $\bar{p}$, there exists an $N \in \mathcal{N}(t)$ with $\operatorname{ess}_{\sup _{N}} p<\mathrm{p}$. For every $\tau \in N$, also by (A.2), $\bar{p}(\tau) \leq \operatorname{ess}_{\sup _{N}} p<\mathrm{p}$. This shows that $\bar{p}$ is upper semicontinuous. The lower semicontinuity of $p$ is proved in the same way.

For Part 2, partition $T$ into $T^{\prime}=\{t: \bar{p}(t)=+\infty\}$ and $T^{\prime \prime}=\{t:-\infty \leq \bar{p}(t)<+\infty\}$. On $T^{\prime}$ obviously $\bar{p}=+\infty \geq p$.

\footnotetext{
${ }^{43}$ If a set $N \neq \emptyset$ is open but $\sigma$-null then $\bar{p}=-\infty<+\infty=\underline{p}$ on $N$. When $T$ has a countable open base, this case can be excluded by removing the largest open set of measure zero, i.e., the union of all open sets of measure zero.
} 
Next, take any sequence of functions $f_{n} \searrow \bar{p}$ on $T^{\prime \prime}$ as $n \nearrow+\infty$, e.g.,

$$
f_{n}(t)= \begin{cases}\bar{p}(t)+\frac{1}{n} & \text { if } \bar{p}(t)>-\infty \\ -n & \text { if } \bar{p}(t)=-\infty\end{cases}
$$

and define

$$
G_{n}:=\left\{t \in T^{\prime \prime}: p(t) \geq f_{n}(t)\right\} .
$$

Fix $n$. For every $t \in T^{\prime \prime}$ there exists an $N \in \mathcal{N}(t)$ with $\sigma\left(G_{n} \cap N\right)=0$ (because nonexistence of such an $N$ would mean that $\left.\bar{p}(t) \geq f_{n}(t)>\bar{p}(t)\right)$. Since such an $N$ can be chosen from the countable open base, there is a countable open cover $\left\{N_{k}: k=1,2, \ldots\right\}$ of $T^{\prime \prime}$ such that $\sigma\left(G_{n} \cap N_{k}\right)=0$ for each $k$. Hence $\sigma\left(G_{n}\right)=0$ for every $n$, and it follows that

$$
\sigma\left\{t \in T^{\prime \prime}: p(t)>\bar{p}(t)\right\}=\sigma\left(\bigcup_{n=1}^{+\infty} G_{n}\right)=0 .
$$

So $p \leq \bar{p}$ a.e. on $T$; and $p \geq \underline{p}$ by a similar argument.

For Part 3, since every $N \in \mathcal{N}(t)$ is $\sigma$-nonnull one has $\operatorname{ess~} \inf _{N} p \leq \operatorname{ess}_{\sup _{N}} p$; and so

$$
\operatorname{ess} \inf _{N} p \leq \underline{p} \leq \bar{p} \leq \underset{N}{\operatorname{ess} \sup _{N} p}
$$

by the definitions (A.1)-A.2).

So when the essential value of $p$ exists everywhere on $T$, it is "automatically" continuous, and it is a variant of $p$.

Corollary A.3. Assume that $T$ has a countable base of open sets which are $\sigma$ nonnull. If dom $\operatorname{ess} p=T$, then $\operatorname{ess} p \in \mathcal{C}(T)$ and $\operatorname{ess} p=p$ almost everywhere on $T$ (w.r.t. $\sigma$ ).

\section{Appendix B. Uniform CONTINUity And inverse CONTINUity}

For a jointly continuous map $M$ on a product of two topological spaces, the implications of compactness of one or both spaces are spelt out (for use in the Proofs of Examples 5.12, 5.13 and 6.2).

Lemma B.1. Assume that $T$ is a (Hausdorff) topological space, $K$ is a metrisable compact with a metric $d_{K}$, and $d_{R}$ is a metric on a set $R$. Then any continuous map $M: T \times K \rightarrow R$ is (jointly) continuous uniformly in the second variable; i.e., for every $t \in T$ and every number $\epsilon>0$ there exist a neighbourhood $H$ of $t$ and a number $\delta>0$ such that for every $t^{\prime}$ and $t^{\prime \prime}$ in $H$ and for every $\mathrm{x}^{\prime}$ and $\mathrm{x}^{\prime \prime}$ in $K$ with $d_{K}\left(\mathrm{x}^{\prime}, \mathrm{x}^{\prime \prime}\right)<\delta$ one has $d_{R}\left(M\left(t^{\prime}, \mathrm{x}^{\prime}\right), M\left(t^{\prime \prime}, \mathrm{x}^{\prime \prime}\right)\right)<\epsilon$.

Comments: 
1. If $T$ is, like $K$, a compact metric space, then so is $T \times K$; in which case every continuous map $M$ is uniformly continuous on $T \times K$ (i.e., for every $\epsilon>0$ there exists a $\delta>0$ such that, for every $t^{\prime}$ and $t^{\prime \prime}$ in $T$ and for every $\mathrm{x}^{\prime}$ and $\mathrm{x}^{\prime \prime}$ in $K$, if $d_{T}\left(t^{\prime}, t^{\prime \prime}\right)<\delta$ and $d_{K}\left(\mathrm{x}^{\prime}, \mathrm{x}^{\prime \prime}\right)<\delta$ then $\left.d_{R}\left(M\left(t^{\prime}, \mathrm{x}^{\prime}\right), M\left(t^{\prime \prime}, \mathrm{x}^{\prime \prime}\right)\right)<\epsilon\right)$. Thisjoint continuity that is uniform in both variables - is obviously stronger than the property established in Lemma B.1.

2. Partial continuity of $M$ in $t$ that holds uniformly in $\mathrm{x}$ is deduced from Lemma B.1 by setting $\mathrm{x}^{\prime}=\mathrm{x}^{\prime \prime}$. This weaker conclusion is stated in, e.g., [19, Lemma IX.3.1].

Proof of Lemma B.1. Suppose contrarily that, for some $t \in T$ and $\epsilon>0$, there exist nets $\left(t_{\mathfrak{n}}^{\prime}\right)$ and $\left(t_{\mathfrak{n}}^{\prime \prime}\right)$ that converge to $t$, and $\left(\mathrm{x}_{\mathfrak{n}}^{\prime}\right)$ and $\left(\mathrm{x}_{\mathfrak{n}}^{\prime \prime}\right)$ with $d_{K}\left(\mathrm{x}_{\mathfrak{n}}^{\prime}, \mathrm{x}_{\mathfrak{n}}^{\prime \prime}\right) \rightarrow 0$, such that

$$
d_{R}\left(M\left(t_{\mathfrak{n}}^{\prime}, \mathrm{x}_{\mathfrak{n}}^{\prime}\right), M\left(t_{\mathfrak{n}}^{\prime \prime}, \mathrm{x}_{\mathfrak{n}}^{\prime \prime}\right)\right) \geq \epsilon \text { for every } \mathfrak{n} \in \mathfrak{N}
$$

(where $\mathfrak{N}$ is the directed set on which the nets are defined). Since $K$ is compact, one can assume (by passing to subnets) that $\left(x_{\mathfrak{n}}^{\prime}\right)$ and $\left(x_{\mathfrak{n}}^{\prime \prime}\right)$ converge, to the same limit $\mathrm{x} \in K$. Then both $M\left(t_{\mathfrak{n}}^{\prime}, \mathrm{x}_{\mathfrak{n}}^{\prime}\right)$ and $M\left(t_{\mathfrak{n}}^{\prime \prime}, \mathrm{x}_{\mathfrak{n}}^{\prime \prime}\right)$ converge to $M(t, \mathrm{x})$; and this contradicts (B.1).

Corollary B.2. If a continuous function $M: T \times[\underline{\mathrm{x}}, \overline{\mathrm{x}}] \rightarrow \mathbb{R}$ is nonincreasing in the second variable (which ranges over a bounded closed interval of $\mathbb{R}$ ), then for every $t \in T$ and $\epsilon>0$ there exist a neighbourhood $H$ of $t$ and $a \delta>0$ such that for every $t^{\prime}$ and $t^{\prime \prime}$ in $H$ and for every $\mathrm{x}^{\prime}$ and $\mathrm{x}^{\prime \prime}$ in $[\underline{\mathrm{x}}, \overline{\mathrm{x}}]$, if $\mathrm{x}^{\prime}<\mathrm{x}^{\prime \prime}+\delta$ then $\epsilon+M\left(t^{\prime}, \mathrm{x}^{\prime}\right)>M\left(t^{\prime \prime}, \mathrm{x}^{\prime \prime}\right)$.

Proof. By Lemma B.1 (applied to $K=[\underline{\mathrm{x}}, \overline{\mathrm{x}}]$ and $R=\mathbb{R}$, both with the usual metric $d(a, b)=|a-b|)$, there exist a neighbourhood $H$ of $t$ and a $\delta>0$ such that $\epsilon+$ $M\left(t^{\prime}, \mathrm{x}^{\prime}\right)>M\left(t^{\prime \prime}, \mathrm{x}^{\prime \prime}\right)$ if $t^{\prime}, t^{\prime \prime} \in H$ and $\mathrm{x}^{\prime \prime} \leq \mathrm{x}^{\prime}<\mathrm{x}^{\prime \prime}+\delta$. In particular, this applies to $\mathrm{x}^{\prime}=\mathrm{x}^{\prime \prime}$; and for the complementary case of $\mathrm{x}^{\prime} \leq \mathrm{x}^{\prime \prime}$ it follows that

$$
\epsilon+M\left(t^{\prime}, \mathrm{x}^{\prime}\right)>M\left(t^{\prime \prime}, \mathrm{x}^{\prime}\right) \geq M\left(t^{\prime \prime}, \mathrm{x}^{\prime \prime}\right)
$$

by the monotonicity of $M\left(t^{\prime \prime}, \cdot\right)$.

When the inverse of $M(t, \cdot)$ exists for each $t$, it is jointly continuous if the domain of $M$ is compact.

Lemma B.3. Assume that both $T$ and $K$ are compact spaces, and $M: T \times K \rightarrow R$ is a (jointly) continuous map into another (Hausdorff) topological space. If for each $t \in T$ the map $M_{t}=M(t, \cdot)$ is invertible, then so is the map

$$
T \times K \ni(t, \mathrm{x}) \mapsto(t, M(t, \mathrm{x})) \in T \times R .
$$

The inverse is defined, on the compact range of (B.2), by

$$
(t, \mathrm{r}) \mapsto\left(t, M_{t}^{-1}(\mathrm{r})\right) \in T \times K
$$

and it is also continuous. Hence the map $(t, \mathrm{r}) \mapsto M_{t}^{-1}(\mathrm{r})$ is continuous.

Proof. With $M_{t}$ invertible, the inverse of (B.2) obviously exists and is given by (B.2). Its continuity, and the compactness of its domain, are special cases of the result stated in, e.g., [27, 5.9.1]. 


\section{REFERENCES}

[1] Bewley, T. (1972): "Existence of equilibria in economies with infinitely many commodities", Journal of Economic Theory, 4, 514-540.

[2] Craven, B. D. (1978): Mathematical programming and control theory. London: Chapman and Hall.

[3] Day, P. W. (1972): "Rearrangement inequalities", Canadian Journal of Mathematics, 24, 930943.

[4] Day, P. W. (1973): "Decreasing rearrangements and doubly stochastic operators", Transactions of the American Mathematical Society, 178, 383-392.

[5] Dellacherie, C., and P. A. Meyer (1978): Probabilities and potential. Amsterdam-New YorkOxford: North-Holland.

[6] Dunford, N., and J. T. Schwartz (1958): Linear operators, Part I: General theory. New York: Interscience.

[7] Horsley, A., and A. J. Wrobel (1986): "The Mackey continuity of the monotone rearrangement", Proceedings of the American Mathematical Society, 97, 626-628.

[8] Horsley, A., and A. J. Wrobel (1987): "The extreme points of some convex sets in the theory of majorization", Proceedings of the Koninklijke Nederlandse Akademie van Wetenschappen, Series A, 90, 171-176.

[9] Horsley, A., and A. J. Wrobel (1988): "Subdifferentials of convex symmetric functions: An application of the Inequality of Hardy, Littlewood and Pólya", Journal of Mathematical Analysis and Applications, 135, 462-475.

[10] Horsley, A., and A. J. Wrobel (1993): "Uninterruptible consumption, concentrated charges, and equilibrium in the commodity space of continuous functions", STICERD Discussion Paper TE/96/300, LSE.

[11] Horsley, A., and A. J. Wrobel (1996): "Efficiency rents of storage plants in peak-load pricing, I: pumped storage", STICERD Discussion Paper TE/96/301, LSE. (This is a fuller version of Ref. [16].)

[12] Horsley, A., and A. J. Wrobel (1999): "Boiteux's solution to the shifting-peak problem and the density form of equilibrium prices in continuous time", STICERD Discussion Paper TE/99/371, LSE (submitted to Economic Theory).

[13] Horsley, A., and A. J. Wrobel (1999): "Efficiency rents of hydroelectric storage plants in continuous-time peak-load pricing", in The current state of economic science (Volume 1, pp. 453-480), ed. by S. B. Dahiya. Spellbound Publications: Rohtak.

[14] Horsley, A., and A. J. Wrobel (1999): "Efficiency rents of storage plants in peak-load pricing, II: hydroelectricity", STICERD Discussion Paper TE/99/372, LSE. (This is a fuller version of Ref. [13].)

[15] Horsley, A., and A. J. Wrobel (2000): "The short-run approach to LRMC pricing for multiple outputs with nondifferentiable costs", STICERD Discussion Paper TE/00/393, LSE (submitted to Journal of Economic Theory).

[16] Horsley, A., and A. J. Wrobel (2001): "Efficiency rents of pumped-storage plants and their uses for operation and investment decisions", Journal of Economic Dynamics and Control, to appear.

[17] Ioffe, A. D., and V. M. Tihomirov (1979): Theory of extremal problems. Amsterdam-New York-Oxford: North-Holland.

[18] Jones, L. E. (1984): "A competitive model of commodity differentiation", Econometrica, 52, $507-530$.

[19] Maurin, K. (1976): Analysis, Part I: Elements. Dordrecht-Boston, MA: Reidel.

[20] Monnier, L. (1983): La tarification de l'électricité en France. Economica: Paris. 
[21] Richard, S. (1989): "A new approach to production equilibria in vector lattices", Journal of Mathematical Economics, 18, 41-56.

[22] Rockafellar, R. T. (1971): "Integrals which are convex functionals, II", Pacific Journal of Mathematics, 39, 439-469.

[23] Rockafellar, R. T. (1974): Conjugate duality and optimization. Philadelphia, PA: SIAM.

[24] Ryff, J. V. (1965): "Orbits of $L^{1}$-functions under doubly stochastic transformations", Transactions of the American Mathematical Society, 117, 92-100.

[25] Ryff, J. V. (1967): Extreme points of some convex subsets of $L^{1}(0,1)$ ", Proceedings of the American Mathematical Society, 18, 1026-1034.

[26] Shiflett, R. C. (1972): "Extreme Markov operators and the orbits of Ryff", Pacific Journal of Mathematics, 40, 201-206.

[27] Sutherland, W. A. (1975): Introduction to metric and topological spaces. Oxford: Oxford University Press.

[28] Tiel, J. van (1984): Convex analysis. Chichester-New York-Brisbane: Wiley.

[29] Tjur, T. (1972): On the mathematical foundations of probability (Lecture Notes 1). Institute of Mathematical Statistics, University of Copenhagen. 